\title{
THE SHARP-INTERFACE APPROACH FOR FLUIDS WITH PHASE CHANGE: RIEMANN PROBLEMS AND GHOST FLUID TECHNIQUES
}

\author{
Christian Merkle ${ }^{1}$ And Christian Rohde ${ }^{2}$
}

\begin{abstract}
Systems of mixed hyperbolic-elliptic conservation laws can serve as models for the evolution of a liquid-vapor fluid with possible sharp dynamical phase changes. We focus on the equations of ideal hydrodynamics in the isothermal case and introduce a thermodynamically consistent solution of the Riemann problem in one space dimension. This result is the basis for an algorithm of ghost fluid type to solve the sharp-interface model numerically. In particular the approach allows to resolve phase transitions sharply, i.e., without artificial smearing in the physically irrelevant elliptic region. Numerical experiments demonstrate the reliability of the method.
\end{abstract}

Mathematics Subject Classification. 35M10, 76T10.

Received August 1st, 2006. Revised April 20, 2007.

\section{INTRODUCTION}

We consider the flow of a compressible fluid that can appear in two phases, lets say, in a liquid and a vapor phase. Mathematical models for phase transition problems split up into so-called sharp-interface models and phase field models. While in the latter approach phase changes are modeled as steep but continuous solutions of the underlying evolution equations the sharp-interface ansatz leads to discontinuous solutions with exactly localized phase transitions.

In this paper we concentrate on a sharp-interface model in one space dimension where phase transitions can be represented as a type of shock wave. More precisely our model consists of the equations of hydrodynamics for an isothermal fluid described by the two conservation laws for density $\rho: \mathbb{R} \times[0, \infty) \rightarrow(0,1 / b)$ and momentum $m:=\rho v: \mathbb{R} \times[0, \infty) \rightarrow \mathbb{R}$ given by

$$
\begin{aligned}
\rho_{t}+m_{x} & =0 \\
m_{t}+\left(\frac{m^{2}}{\rho}+p(\rho)\right)_{x} & =0 \quad \text { in } \mathbb{R} \times(0, \infty) .
\end{aligned}
$$

Keywords and phrases. Dynamical phase transitions in compressible media, van-der-Waals pressure, kinetic relations, Riemann solver, ghost fluid approach.

1 Abteilung für Angewandte Mathematik, lbert-Ludwigs-Universität Freiburg, Hermann-Herder Str. 10 , 79104 Freiburg, Germany. christian@mathematik.uni-freiburg.de

2 Institut für Angewandte Mathematik und Numerische Simulation, Universität Stuttgart, Pfaffenwaldring 57, 70569 Stuttgart, Germany. 

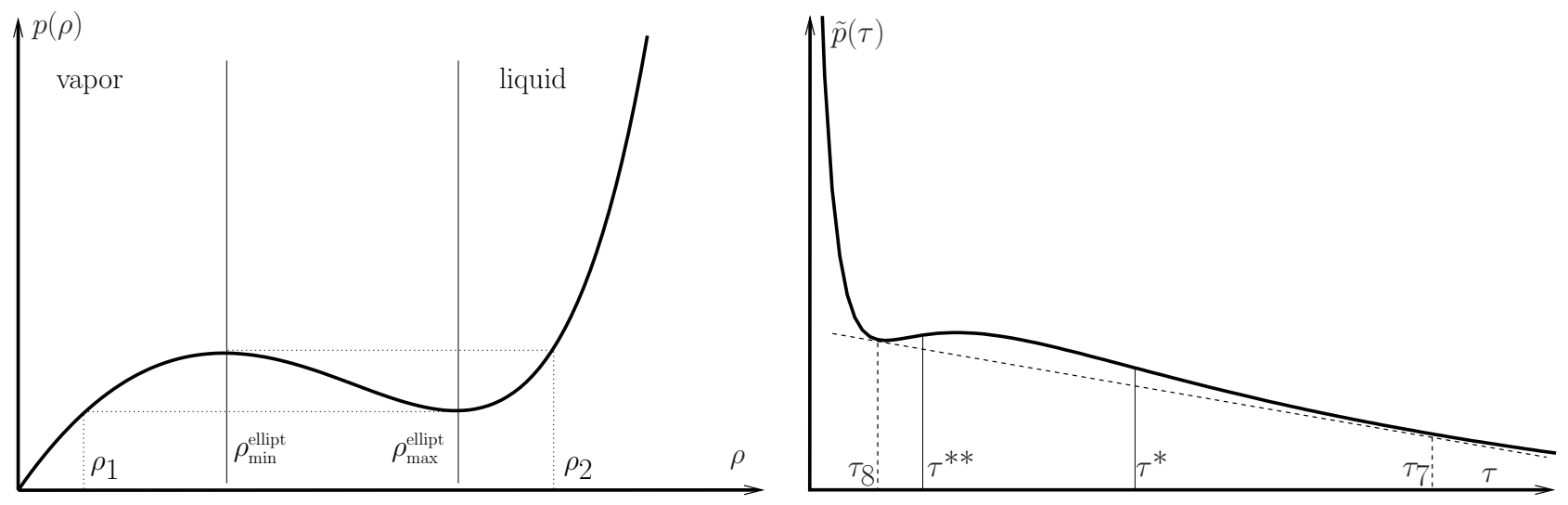

Figure 1. Graph of the van-der-Waals pressure function $p=p(\rho)$ and the associated Lagrangian pressure $\tilde{p}=\tilde{p}(\tau)$ with $\tilde{p}(\tau)=p(1 / \tau)$ for sufficiently low temperature.

We consider the Cauchy problem for (1) and, for initial density $\rho_{0}: \mathbb{R} \rightarrow(0,1 / b)$ and velocity $v_{0}: \mathbb{R} \rightarrow \mathbb{R}$, enforce the conditions

$$
\rho(., 0)=\rho_{0}, \quad m(., 0)=\rho_{0} v_{0} \quad \text { in } \mathbb{R} .
$$

Here $b$ is a positive number and for the smooth pressure function $p:(0,1 / b) \rightarrow(0, \infty)$ we suppose that there are numbers $\rho_{\min }^{\text {ellipt }}, \rho_{\max }^{\text {ellipt }} \in(0,1 / b)$ with $\rho_{\min }^{\text {ellipt }}<\rho_{\max }^{\text {ellipt }}$ such that $p$ is monotone increasing in the admissible range

and monotone decreasing in the elliptic region

$$
\mathcal{A}:=\left(0, \rho_{\min }^{\text {ellipt }}\right) \cup\left(\rho_{\max }^{\text {ellipt }}, 1 / b\right)
$$

$$
(0,1 / b) \backslash \mathcal{A}=\left[\rho_{\min }^{\text {ellipt }}, \rho_{\max }^{\text {ellipt }}\right] .
$$

We refer to Figure 1 for the typical graph of $p$ and to Section 1 for precise assumptions. On this general level it is only important that the non-monotone shape of $p$ allows to define phases. A state $(\rho, \rho v)$ in the physical state space $\mathcal{A} \times \mathbb{R}$ is called a liquid (vapor) state if $\rho \in\left(0, \rho_{\min }^{\text {ellipt }}\right]\left(\rho \in\left[\rho_{\max }^{\text {ellipt }}, 1 / b\right)\right)$ holds. As a consequence of the non-monotone shape of $p$ the first-order system (1) is hyperbolic for states if and only if the states are liquid or vapor. The system (or more exactly its linearized version) is elliptic for density values in $(0,1 / b) \backslash \mathcal{A}$. Altogether we obtain a mixed-type system. Mixed-type systems are frequently used as models for phase transitions in compressible media ( $c f .[1,12])$.

Our ultimate goal is to design a numerical method to solve the multidimensional version of the sharp-interface model (1). In this paper we restrict ourselves to the 1D-case such that curvature effects play no role. Usually in many phase transition and phase separation problems the correct treatment of curvature effects is one of the main challenges. We would like to stress that here - due to the interaction of flow and phase transition effects - even the 1D-case is by far not trivial. In the rest of the introduction we give an outline of the paper's content and indicate the specific difficulties in this sharp-interface model.

The basis of our numerical method is the exact treatment of the Riemann problem for the conservation law (1), that is the special initial data choice

$$
\left(\begin{array}{c}
\rho \\
m
\end{array}\right)(x, 0)=\left\{\begin{array}{c}
\left(\begin{array}{c}
\rho_{L} \\
m_{L}:=\rho_{L} v_{L}
\end{array}\right): x<0 \\
\left(\begin{array}{c}
\rho_{R} \\
m_{R}:=\rho_{R} v_{R}
\end{array}\right): x>0
\end{array} \quad\left(\rho_{L}, \rho_{R} \in \mathcal{A}, v_{L}, v_{R} \in \mathbb{R}\right) .\right.
$$

System (1) has the form of a conservation law, however the standard solution theory for hyperbolic conservation laws does not apply (see e.g. $[11,23,36]$ ). This is first due to the fact that the elliptic region separates the state 
space into two disjoint sets where (1) is hyperbolic. Secondly, the system is not genuinely nonlinear in the vapor phase anymore which requires to build Riemann patterns with attached-wave structures. Moreover, phase transitions - static as well as dynamical ones - are naturally modeled as shock waves and thus appear automatically as sharp interfaces. But they are (partly) not Laxian-type shock waves but of the nonclassical undercompressive type. Let us also stress as a striking property of the system (1) that the Clausius-Duhem inequality is not enough to ensure the unique solvability of the Riemann problem (see [23]). To overcome these difficulties we follow the approach developed by LeFloch and co-workers $[5,17,24]$ which relies on the concept of the kinetic relation $[1,39,40]$. The main existence result for a thermodynamically admissible Riemann problem solution is then presented at the end of Section 3 (Thm. 3.6). The preceding sections serve to introduce the model (Sect. 1) and to present a careful analysis for all possible wave types and their thermodynamical admissibility including shock waves, rarefaction waves, attached waves and phase transitions (Sect. 2). In this context we mention the publications $[9,10,16,30]$ on the existence and stability of solutions for the Riemann problem for related systems.

A well-developed class of methods to solve the Euler equations for compressible one-phase fluids is provided by upwind finite volume schemes based on approximate Riemann solvers. In Section 4 we propose a similar class of methods for the Cauchy problem (1), (2). However standard averaging techniques can not be used in our case since this - due to the non convex state space - might lead to approximate cell average states in the elliptic region.

To circumvent this difficulty we suggest a ghost-fluid type algorithm, motivated but different from the algorithm in the seminal paper [13] (see $[2,4,14,20,26]$ for other applications). The crucial analytical basis of the ghost fluid algorithm is the Riemann solver which has been developed in the first part of the paper (cf. Sects. 2 and 3). Finally, we test the algorithm on problems with known exact solution and report on a number of numerical experiments. We have not been able to prove rigorously the convergence of our algorithm as the mesh parameter tends to zero but we present a convergence result for simple phase transition solutions with Proposition 4.5. In forthcoming papers we extend the approach to multiple space dimensions (see in particular [27]) and address curvature effects and the non-isothermal situation.

Another direct numerical method for the mixed-type Cauchy problem (1), (2) apart from the one presented here is not known to us. However we have to refer to several related publications. A different approach to avoid averaging effects is the DEM-method due to Abgrall and Saurel [3] which has been used in [21] to simulate evaporation fronts. For the numerical solution of the sharp interface model with additional kinetic relations there has been proposed the Glimm-Scheme [8,22], front-tracking schemes [41], artificial dissipation methods [25] and a level-set scheme $[18,28]$. The latter one is different from the one presented here since the authors extend the kinetic relation to the whole computational domain. Recently Chalons has developed a new deterministic method to treat nonclassical shocks with kinetic relations in hyperbolic equations [7].

\section{BASIC PROPERTIES OF THE MIXED-TYPE SYSTEM}

In this section we detail all assumptions on the system (1) and introduce fundamental notations. Furthermore we review the spectral properties of the nonlinear flux in (1) and the structure of the associated characteristic fields.

Assumption 1.1 (Pressure function).

(i) The function $p \in C^{2}((0,1 / b),(0, \infty))$ is supposed to satisfy

$$
\begin{array}{cll}
p^{\prime}(\rho)>0, p^{\prime \prime}(\rho)<0 & \text { for } & \rho \in\left(0, \rho_{\text {min }}^{\text {ellipt }}\right), \\
p^{\prime}(\rho)>0, p^{\prime \prime}(\rho)>0 & \text { for } & \rho \in\left(\rho_{\text {max }}^{\text {ellipt }}, 1 / b\right), \\
p^{\prime}(\rho)<0 & \text { for } & \rho \in\left(\rho_{\min }^{\text {ellipt }}, \rho_{\text {max }}^{\text {ellipt }}\right) .
\end{array}
$$


(ii) Furthermore, we have

$$
\lim _{\rho \rightarrow 0} p(\rho)=\lim _{\rho \rightarrow 0} p^{\prime}(\rho) \rho^{2}=0, \quad \lim _{\rho \nearrow 1 / b} p(\rho)=\infty .
$$

The typical shape of such a pressure function is shown in Figure 1 (left graph). Due to the fundamental laws of thermodynamics the associated energy density function $W \in C^{1}(0,1 / b)$ is given up to an unimportant free constant by the relation

$$
p(\rho)=\rho W^{\prime}(\rho)-W(\rho)
$$

Furthermore, from (4) it is clear (see also Fig. 1) that there are unique densities $\rho_{1} \in\left(0, \rho_{\text {min }}^{\text {ellipt }}\right)$ and $\rho_{2} \in$ $\left(\rho_{\max }^{\text {ellipt }}, 1 / b\right)$ such that we have

$$
p\left(\rho_{1}\right)=p\left(\rho_{\max }^{\text {ellipt }}\right) \text { and } p\left(\rho_{2}\right)=p\left(\rho_{\min }^{\text {ellipt }}\right) .
$$

The Maxwell states are defined as the two points $\rho_{\text {vapor }}^{M}$ and $\rho_{\text {liquid }}^{M}$ such that

$$
W^{\prime}\left(\rho_{\text {vapor }}^{M}\right)=W^{\prime}\left(\rho_{\text {liquid }}^{M}\right)=\frac{W\left(\rho_{\text {liquid }}^{M}\right)-W\left(\rho_{\text {vapor }}^{M}\right)}{\rho_{\text {liquid }}^{M}-\rho_{\text {vapor }}^{M}}
$$

That means the straight line connecting $\left(\rho_{\text {vapor }}^{M}, W\left(\rho_{\text {vapor }}^{M}\right)\right)$ and $\left(\rho_{\text {liquid }}^{M}, W\left(\rho_{\text {liquid }}^{M}\right)\right)$ has the same slope as $W$ at $\rho_{\text {vapor }}^{M}$ and $\rho_{\text {liquid }}^{M}$ (cf. Fig. 1). A short calculation using (6) and (8) leads to the property

$$
p\left(\rho_{\text {vapor }}^{M}\right)=p\left(\rho_{\text {liquid }}^{M}\right) .
$$

In our Riemann solution for (1), (3) later on static equilibria occur if and only if the connecting states are the Maxwell states.

Remark 1.2. For appropriate choices of the constant temperature $T>0$ and constants $a, R>0$ a pressure function satisfying (4) and (5) can be realized as the van-der-Waals function

$$
p(\rho)=\frac{\rho R T}{1-b \rho}-a \rho^{2} .
$$

Actually all figures and numerical calculations in the following are performed with the constants $a=3, b=$ $1 / 3, R=8, T=98 / 300$.

The eigenvalues and corresponding eigenvectors of the Jacobian of the flux in (1) are

$$
\lambda_{1 / 2}(\rho, m)=\frac{m}{\rho} \mp \sqrt{p^{\prime}(\rho)}, \quad \vec{r}_{1 / 2}(\rho, m)=\left(\begin{array}{c}
1 \\
\frac{m}{\rho} \mp \sqrt{p^{\prime}(\rho)}
\end{array}\right) .
$$

We observe that the system (1) is hyperbolic for states $(\rho, m) \in \mathcal{A} \times \mathbb{R}$, if and only if $p^{\prime}(\rho)>0$ holds, that is, if and only if the state is a liquid or vapor state.

We compute for $(\rho, m) \in \mathcal{A} \times \mathbb{R}$ the characteristic fields of the flux and get the following expression independent of momentum:

$$
\nabla \lambda_{1 / 2}(\rho, m) \cdot \vec{r}_{1 / 2}(\rho, m)=\mp\left(\frac{p^{\prime \prime}(\rho)}{2 \sqrt{p^{\prime}(\rho)}}+\frac{\sqrt{p^{\prime}(\rho)}}{\rho}\right)=: \Lambda_{\mp}\left(\frac{1}{\rho}\right) .
$$


Rewriting the last equations in terms of $\tau=1 / \rho$ we obtain

$$
\Lambda_{\mp}(\tau)=\mp\left(\frac{2 \tau^{2} \tilde{p}^{\prime}(\tau)+\tau^{3} \tilde{p}^{\prime \prime}(\tau)}{2 \sqrt{-\tilde{p}^{\prime}(\tau)}}+\tau^{2} \sqrt{-\tilde{p}^{\prime}(\tau)}\right) .
$$

Here we used the Lagrangian pressure $\tilde{p}:(b, \infty) \rightarrow(0, \infty)$ defined by

$$
\tilde{p}(\tau):=p(1 / \tau) \quad(\tau \in(b, \infty))
$$

We refer to Figure 1, right graph, for a visualization of $\tilde{p}$. From (4), (5) we see that $\tilde{p}^{\prime}\left(1 / \rho_{\min }^{\text {ellipt }}\right)=0$ and $\lim _{\tau \rightarrow \infty} \tilde{p}^{\prime}(\tau)=0$ hold. Therefore, there exists an inflection point $\tau^{*} \in\left(1 / \rho_{\min }^{\text {ellipt }}, \infty\right)$ and another inflection point $\tau^{* *}$ of the function $\tilde{p}$ for which

is located in the elliptic region. Define also

$$
\rho^{* *}:=\frac{1}{\tau^{* *}}
$$

$$
\rho^{*}:=\frac{1}{\tau^{*}} \in\left(0, \rho_{\min }^{\text {ellipt }}\right)
$$

Note, that $\tilde{p}$ is convex in $\left(b, \tau^{* *}\right) \cup\left(\tau^{*}, \infty\right)$ and concave in $\left(\tau^{* *}, \tau^{*}\right)$. We refer to Figure 1 for the graph of $\tilde{p}$.

Returning to (12) we see that $\Lambda_{\mp}=\Lambda_{\mp}(\tau)$ vanishes for $\tau \in(b, \infty)$ if and only if we have

$$
\frac{1}{2}\left(2 \tau^{2} \tilde{p}^{\prime}(\tau)+\tau^{3} \tilde{p}^{\prime \prime}(\tau)\right)-\tau^{2} \tilde{p}^{\prime}(\tau)=\tilde{p}^{\prime \prime}(\tau)=0
$$

Thus both characteristic fields of the flux in (1) fail to be genuinely nonlinear for liquid states with $\rho=\rho^{*}$.

\section{BASIC ELEMENTARY WAVES AND THERMODYNAMICAL ADMISSIBILITY}

In this section we consider all elementary waves which we need in the construction of the weak solution for the Riemann problem (1), (3) in Section 3 below. We start with shock waves and general phase boundaries. To detect the physically admissible shock waves we analyze the entropy dissipation function and to solve the problem of the failure of genuine nonlinearity we carefully consider the location of associated end states in the phase plane. Finally we discuss rarefaction waves resp. attached waves and - last but not least - non-Laxian shock waves that satisfy a given kinetic relation.

\subsection{Rankine-Hugoniot conditions, shock waves, and phase transitions}

Basic elementary waves for the solution of the Riemann problem for (1) are shock waves. A shock wave ${ }^{1}$ with speed $s \in \mathbb{R}$ connecting a state $\left(\rho_{l}, m_{l}\right) \in \mathcal{A} \times \mathbb{R}$ with a state $\left(\rho_{r}, m_{r}\right) \in \mathcal{A} \times \mathbb{R}$ (we also write $\left.\left(\rho_{l}, m_{l}\right) \stackrel{s}{\rightarrow}\left(\rho_{r}, m_{r}\right)\right)$ is a discontinuous function of the type

$$
\left(\begin{array}{c}
\rho \\
m
\end{array}\right)=\left(\begin{array}{c}
\rho(x, t) \\
m(x, t)
\end{array}\right)= \begin{cases}\left(\rho_{l}, m_{l}\right)^{T} & : x<s t \\
\left(\rho_{r}, m_{r}\right)^{T} & : x>s t\end{cases}
$$

that fulfills the Rankine-Hugoniot jump conditions

$$
s \llbracket \rho \rrbracket=\llbracket m \rrbracket \quad \text { and } \quad s \llbracket m \rrbracket=\llbracket \frac{m^{2}}{\rho}+p(\rho) \rrbracket .
$$

\footnotetext{
${ }^{1}$ The notation is sloppy since usually discontinuous traveling waves satisfying the Rankine-Hugoniot conditions but having zero entropy dissipation are not called shock waves. For the sake of simplicity we include them here.
} 
In (14) is and henceforth will be used the notation $\llbracket a \rrbracket:=a_{l}-a_{r}$ for some general variable $a$. The conditions (14) imply in particular that (13) is a weak solution of $(1)$.

Now, fixing a state $\left(\rho_{l}, m_{l}\right) \in \mathcal{A} \times \mathbb{R}$ we can determine the set of states $\left(\rho_{r}, m_{r}\right)$ hat can be connected to $\left(\rho_{l}, m_{l}\right) \in \mathcal{A} \times \mathbb{R}$ by a shock wave, the so called Rankine-Hugoniot set for $\left(\rho_{l}, m_{l}\right)$. For first-order conservation laws the Rankine-Hugoniot set can be characterized easily provided there is an open set containing $\left(\rho_{l}, m_{l}\right)$ where the system is hyperbolic. In our case this is true within each phase and we have the following result (see e.g. [15], Thm. 4.1).

Theorem 2.1. Let $\left(\rho_{l}, m_{l}\right) \in \mathcal{A} \times \mathbb{R}$. There exists a number $\varepsilon_{0}>0$, functions $\Phi_{k}:\left(-\varepsilon_{0}, \varepsilon_{0}\right) \rightarrow \mathcal{A} \times \mathbb{R}$, $k=1,2$, and a neighborhood of $\left(\rho_{l}, m_{l}\right)$ such that the set $\left\{\Phi_{k}(\varepsilon) \mid \varepsilon \in\left(-\varepsilon_{0}, \varepsilon_{0}\right), k=1,2\right\}$ coincides with the Rankine-Hugoniot set for $\left(\rho_{l}, m_{l}\right)$ in this neighborhood. Moreover we have for $\varepsilon \in\left(-\varepsilon_{0}, \varepsilon_{0}\right)$ and $k=1,2$

$$
\Phi_{k}(\epsilon)=\left(\rho_{l}, m_{l}\right)^{T}+\epsilon \vec{r}_{k}\left(\rho_{l}, m_{l}\right)+O\left(\epsilon^{2}\right)
$$

and

$$
s=s\left(\left(\rho_{l}, m_{l}\right), \Phi_{k}(\epsilon)\right)=\lambda_{k}\left(\rho_{l}, m_{l}\right)+O(\epsilon) .
$$

According to the numbering of the curves in Theorem 2.1 we speak of $k$-shock waves. In the case of a 1 -shock wave a specific parameterization as in (15) is given by

$$
\left(\begin{array}{c}
\rho_{r} \\
m_{r}
\end{array}\right)=\Phi_{1}(\epsilon)=\left(\begin{array}{c}
\rho_{l} \\
m_{l}
\end{array}\right)+\epsilon\left(\begin{array}{c}
1 \\
\frac{m_{l}}{\rho_{l}}-\sqrt{p^{\prime}\left(\rho_{l}\right)}
\end{array}\right)+O\left(\epsilon^{2}\right) .
$$

It follows for $\varepsilon \in\left(-\varepsilon_{0}, \varepsilon_{0}\right)$

$$
\frac{v_{r}-v_{l}}{\rho_{r}-\rho_{l}}=-\frac{\sqrt{p^{\prime}\left(\rho_{l}\right)}}{\rho_{r}}+O(\epsilon)
$$

Therefore we have

$$
v_{r}-v_{l} \geq 0(\leq 0) \text { for } \rho_{r} \leq \rho_{l} \quad\left(\rho_{r} \geq \rho_{l}\right) .
$$

Returning to the global situation system (14) consists of two equations with three unknowns $\rho_{r}, v_{r}$ and $s$. These can be reformulated for $v_{r}$ and $s$ depending on $\rho_{r}$ :

$$
\begin{aligned}
v_{r}\left(\rho_{r}\right) & =v_{l} \mp \sqrt{\frac{\rho_{r}-\rho_{l}}{\rho_{r} \rho_{l}}\left(p\left(\rho_{r}\right)-p\left(\rho_{l}\right)\right)}, \\
s\left(\rho_{r}\right) & =v_{l} \mp \sqrt{\frac{\rho_{r}}{\rho_{l}} \frac{p\left(\rho_{r}\right)-p\left(\rho_{l}\right)}{\rho_{r}-\rho_{l}}}=v_{r} \mp \sqrt{\frac{\rho_{l}}{\rho_{r}} \frac{p\left(\rho_{r}\right)-p\left(\rho_{l}\right)}{\rho_{r}-\rho_{l}} .}
\end{aligned}
$$

We observe that globally the Rankine-Hugoniot set consists of two curves also called $k$-shock wave curves. Comparing (18) with the local parameterization (17) we conclude: The 1-shock wave curve is given by

$$
v_{r}-v_{l}=\left\{\begin{array}{l}
+\sqrt{\frac{\rho_{r}-\rho_{l}}{\rho_{l} \rho_{r}}\left(p\left(\rho_{r}\right)-p\left(\rho_{l}\right)\right)}: \rho_{r} \leq \rho_{l}, \\
-\sqrt{\frac{\rho_{r}-\rho_{l}}{\rho_{l} \rho_{r}}\left(p\left(\rho_{r}\right)-p\left(\rho_{l}\right)\right)}: \rho_{r} \geq \rho_{l} .
\end{array}\right.
$$

Equivalently, 2-shock wave curve is given by

$$
v_{r}-v_{l}=\left\{\begin{array}{l}
-\sqrt{\frac{\rho_{r}-\rho_{l}}{\rho_{l} \rho_{r}}\left(p\left(\rho_{r}\right)-p\left(\rho_{l}\right)\right)}: \rho_{r} \leq \rho_{l}, \\
+\sqrt{\frac{\rho_{r}-\rho_{l}}{\rho_{l} \rho_{r}}\left(p\left(\rho_{r}\right)-p\left(\rho_{l}\right)\right)}: \rho_{r} \geq \rho_{l} .
\end{array}\right.
$$


Remark 2.2. The parameterizations of the Rankine-Hugoniot sets (20) and (21) with $v_{r}$ seen as function of the right-hand state $\rho_{r} \in \mathcal{A}$ satisfy the monotonicity property

$$
\frac{\mathrm{d} v_{r}}{\mathrm{~d} \rho_{r}}\left(\rho_{r}\right)<0(>0) \text { for a 1-shock wave (a 2-shock wave) }
$$

To prove this for a 1-shock wave consider

$$
\frac{\mathrm{d} v_{r}}{\mathrm{~d} \rho_{r}}\left(\rho_{r}\right)= \pm\left(\frac{\rho_{r}-\rho_{l}}{\rho_{r}^{2}}\right) \underbrace{\left(\sqrt{\frac{\rho_{r}-\rho_{l}}{\rho_{l} \rho_{r}}\left(p\left(\rho_{r}\right)-p\left(\rho_{l}\right)\right)}\right)^{-\frac{1}{2}}\left(\frac{p\left(\rho_{r}\right)-p\left(\rho_{l}\right)}{\rho_{r}-\rho_{l}}+\frac{\rho_{r}}{\rho_{l}} p^{\prime}\left(\rho_{r}\right)\right)}_{>0},
$$

where the $+\operatorname{sign}$ is valid if $\rho_{r} \leq \rho_{l}$ and the $-\operatorname{sign}$ if $\rho_{r}>\rho_{l}$. Hence, $\frac{\mathrm{d} v_{r}}{\mathrm{~d} \rho_{r}}\left(\rho_{r}\right)<0$ for a 1-shock wave. For a 2 -shock wave the calculation works the same replacing " \pm ” by “干”.

\subsection{Entropy dissipation and characteristic structure}

Physically meaningful shock waves $\left(\rho_{l}, m_{l}\right) \stackrel{s}{\rightarrow}\left(\rho_{r}, m_{r}\right)$ also have to satisfy the entropy dissipation inequality or Clausius-Duhem condition, that is,

$$
s \llbracket E(\rho, v) \rrbracket-\llbracket F(\rho, v) \rrbracket \leq 0 .
$$

The entropy $E:(0,1 / b) \times \mathbb{R} \longrightarrow \mathbb{R}$ and the entropy flux $F:(0,1 / b) \times \mathbb{R} \longrightarrow \mathbb{R}$ are given by

$$
E(\rho, v)=\frac{1}{2} \rho v^{2}+W(\rho), \quad F(\rho, v)=v\left(\frac{1}{2} \rho v^{2}+W(\rho)+p(\rho)\right)
$$

where $W=W(\rho)$ is the free energy of the system $(c f .(6))$. A shock wave that satisfies (22) is called entropydissipative.

Later on in this section we need a reformulation of the entropy dissipation which we derive now. We get from (14), (22), (23), and (6)

$$
\begin{aligned}
s \llbracket E(\rho, v) \rrbracket-\llbracket F(\rho, v) \rrbracket= & s\left(\frac{1}{2} \rho_{l} v_{l}^{2}+W\left(\rho_{l}\right)-\frac{1}{2} \rho_{r} v_{r}^{2}-W\left(\rho_{r}\right)\right) \\
& -v_{l}\left(p\left(\rho_{l}\right)+\frac{1}{2} \rho_{l} v_{l}^{2}+W\left(\rho_{l}\right)\right)+v_{r}\left(p\left(\rho_{r}\right)+\frac{1}{2} \rho_{r} v_{r}^{2}+W\left(\rho_{r}\right)\right) \\
= & -\frac{j}{2}\left(v_{l}^{2}-v_{r}^{2}\right)-s \llbracket p \rrbracket-j \llbracket W^{\prime}(\rho) \rrbracket \\
= & -\frac{j}{2}\left(\llbracket v \rrbracket\left(v_{l}+v_{r}-2 s\right)+\llbracket W^{\prime}(\rho) \rrbracket\right) .
\end{aligned}
$$

Here we used $j:=\rho_{l}\left(v_{l}-s\right)=\rho_{r}\left(v_{r}-s\right)$. A straightforward computation using (18), (19) leads for $\rho_{l} \neq \rho_{r}$ to

$$
s \llbracket E(\rho, m) \rrbracket-\llbracket F(\rho, m) \rrbracket=\frac{-j \llbracket \rho \rrbracket^{3}}{2 \rho_{l} \rho_{r}}\left(\left(\rho_{l}+\rho_{r}\right) \frac{\llbracket W(\rho) \rrbracket}{\llbracket \rho \rrbracket^{3}}-\frac{\rho_{l} W^{\prime}\left(\rho_{l}\right)+\rho_{r} W^{\prime}\left(\rho_{r}\right)}{\llbracket \rho \rrbracket^{2}}\right)=: \frac{-j \llbracket \rho \rrbracket^{3}}{2 \rho_{l} \rho_{r}} \Delta\left(\rho_{l}, \rho_{r}\right) .
$$


Moreover, defining $\tilde{\Delta}\left(\tau_{l}, \tau_{r}\right):=\Delta\left(\frac{1}{\tau_{l}}, \frac{1}{\tau_{r}}\right)$ and using relation (6) yields

$$
\begin{aligned}
-\frac{\llbracket \rho \rrbracket^{3}}{2 \rho_{l} \rho_{r}} \Delta\left(\rho_{l}, \rho_{r}\right) & =-\frac{\llbracket \rho \rrbracket^{3}}{2 \rho_{l} \rho_{r}} \tilde{\Delta}\left(\tau_{l}, \tau_{r}\right) \\
& =\frac{\tilde{p}\left(\tau_{l}\right)+\tilde{p}\left(\tau_{r}\right)}{2}\left(\tau_{r}-\tau_{l}\right)-\int_{\tau_{l}}^{\tau_{r}} \tilde{p}(s) \mathrm{d} s \\
& =\int_{\tau_{l}}^{\tau_{r}}\left(\tilde{p}\left(\tau_{l}\right)+\frac{\tilde{p}\left(\tau_{r}\right)-\tilde{p}\left(\tau_{l}\right)}{\tau_{r}-\tau_{l}}\left(s-\tau_{l}\right)-\tilde{p}(s)\right) \mathrm{d} s .
\end{aligned}
$$

This formulation allows a nice geometrical interpretation to determine the sign and the zero level set of the function $\Delta$. Let $\tau_{l}, \tau_{r} \in(b, \infty)$. Then we have $\tilde{\Delta}\left(\tau_{l}, \tau_{r}\right)=0$ if and only if

$$
\int_{\tau_{l}}^{\tau_{r}}\left(\tilde{p}\left(\tau_{l}\right)+\frac{\tilde{p}\left(\tau_{r}\right)-\tilde{p}\left(\tau_{l}\right)}{\tau_{r}-\tau_{l}}\left(s-\tau_{l}\right)-\tilde{p}(s)\right) \mathrm{d} s=0,
$$

i.e. if the (signed) area between the straight line connecting $\tilde{p}\left(\tau_{l}\right)$ and $\tilde{p}\left(\tau_{r}\right)$ and the graph of $\tilde{p}$ is zero. As we are interested in the zero dissipation level set we define the set

$$
\Gamma_{\Delta}:=\left\{\left(\rho_{l}, \rho_{r}\right) \in(0,1 / b)^{2} \mid \Delta\left(\rho_{l}, \rho_{r}\right)=0, \rho_{l} \neq \rho_{r}\right\} \cup\left(\rho^{*}, \rho^{*}\right) \cup\left(\rho^{* *}, \rho^{* *}\right) .
$$

Note that the function $\Delta$ is only defined for $\rho_{l} \neq \rho_{r}$ but there is a continuous extension since

$$
\lim _{\tilde{\rho} \rightarrow \rho} \Delta(\tilde{\rho}, \rho)=-\frac{1}{6}\left(p^{\prime \prime}(\rho)+2 p^{\prime}(\rho)\right)=-\frac{1}{6} \tau^{4} \tilde{p}_{\tau \tau}(\tau) .
$$

The latter expression vanishes exactly for $\tau=\tau^{*}, \tau^{* *}$ and therefore we added the points $\left(\rho^{*}, \rho^{*}\right)$ and $\left(\rho^{* *}, \rho^{* *}\right)$ in definition (27).

We observe that $\Gamma_{\Delta}$ is symmetric with respect to the axis $\{(\rho, \rho) \mid \rho \in(0,1 / b)\}$ and also deduce from (8)

$$
\left(\rho_{\text {vapor }}^{M}, \rho_{\text {liquid }}^{M}\right),\left(\rho_{\text {liquid }}^{M}, \rho_{\text {vapor }}^{M}\right) \in \Gamma_{\Delta} .
$$

Moreover, the set $\Gamma_{\Delta}$ is a closed curve in $(0,1 / b)^{2}$, which follows from the geometrical interpretation (26) and Assumption 1.1 on the pressure function $p$.

For the further analysis it is important to classify shock waves according to the following standard system. For $k \in\{1,2\}$ a $k$-shock wave is called a Laxian (or Lax-compressive) shock wave if the condition

$$
\lambda_{k}\left(\rho_{l}, m_{l}\right) \geq s \geq \lambda_{k}\left(\rho_{r}, m_{r}\right)
$$

is satisfied. If one of the relations in (29) holds with equality the corresponding shock wave is called characteristic. Furthermore, in the context of this paper a shock wave is called an undercompressive shock wave if

$$
\lambda_{2}\left(\rho_{l}, m_{l}\right)>s>\lambda_{1}\left(\rho_{r}, m_{r}\right) \text { and } \lambda_{2}\left(\rho_{r}, m_{r}\right)>s>\lambda_{1}\left(\rho_{l}, m_{l}\right)
$$

is satisfied. The definitions are illustrated in Figure 2. In the framework of the first-order system (1) it is natural to view phase jumps as shock waves: a shock wave $\left(\rho_{l}, m_{l}\right) \stackrel{s}{\rightarrow}\left(\rho_{r}, m_{r}\right)$ is called a phase transition, if the left and the right states are located in different phases (see Fig. 1). In this case we also use the notation $\left(\rho_{l}, m_{l}\right) \stackrel{p t}{\rightarrow}\left(\rho_{r}, m_{r}\right)$. We will see that in our case undercompressive shock waves will only appear as phase 

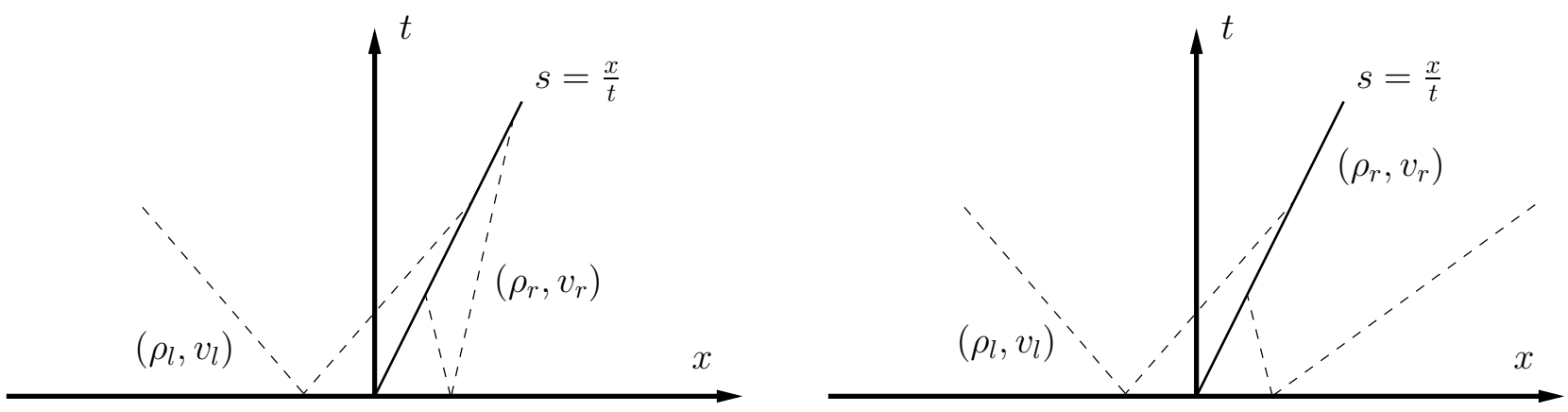

FiguRE 2. Laxian 2-shock wave (left) and undercompressive shock wave (right). Shock lines for shocks with speed $s$ and some characteristic curves (dashed lines) in the $(x, t)$-half plane.

transitions while Laxian waves can be purely hydrodynamical shock waves or phase transitions. Equation (29) is - using $\tau=1 / \rho$ and $\tilde{p}(\tau)=p(1 / \tau)$ - equivalent to

$$
\tilde{p}_{\tau}\left(\tau_{l}\right) \leq \frac{\tilde{p}\left(\tau_{r}\right)-\tilde{p}\left(\tau_{l}\right)}{\tau_{r}-\tau_{l}} \leq \tilde{p}_{\tau}\left(\tau_{r}\right)
$$

for Laxian 1-shock waves and

$$
\tilde{p}_{\tau}\left(\tau_{l}\right) \geq \frac{\tilde{p}\left(\tau_{r}\right)-\tilde{p}\left(\tau_{l}\right)}{\tau_{r}-\tau_{l}} \geq \tilde{p}_{\tau}\left(\tau_{r}\right)
$$

for Laxian 2-shock waves.

In the hyperbolic case (in each phase in our case), it can be shown that shock waves (13) that satisfy the Lax condition (29) are entropy solutions, i.e. (22) holds. To account for the possible difference between Laxian shocks and entropy dissipative Laxian shocks in the two-phase case we define the following sets:

$$
\begin{aligned}
& \mathcal{M}^{1}:=\left\{\left(\rho_{l}, \rho_{r}\right) \in \mathcal{A}^{2} \mid \exists\left(m_{l}, m_{r}, s\right) \in \mathbb{R}^{3}:(14) \text { and (31) hold }\right\} \\
& \mathcal{M}^{2}:=\left\{\left(\rho_{l}, \rho_{r}\right) \in \mathcal{A}^{2} \mid \exists\left(m_{l}, m_{r}, s\right) \in \mathbb{R}^{3}:(14) \text { and (32) hold }\right\}
\end{aligned}
$$

The sets $\mathcal{M}^{1}$ and $\mathcal{M}^{2}$ are displayed in Figure 3. Since we are interested in entropy dissipative Laxian shock waves we introduce the subsets

$$
\begin{aligned}
& \mathcal{M}_{\text {diss }}^{1}:=\left\{\left(\rho_{l}, \rho_{r}\right) \in \mathcal{A}^{2} \mid \exists\left(m_{l}, m_{r}, s\right) \in \mathbb{R}^{3}:(14),(31) \text { and (22) hold }\right\} \\
& \mathcal{M}_{\text {diss }}^{2}:=\left\{\left(\rho_{l}, \rho_{r}\right) \in \mathcal{A}^{2} \mid \exists\left(m_{l}, m_{r}, s\right) \in \mathbb{R}^{3}:(14),(32) \text { and (22) hold }\right\} .
\end{aligned}
$$

Thus $\mathcal{M}_{\text {diss }}^{1}$ and $\mathcal{M}_{\text {diss }}^{2}$ are bounded by (parts of) the boundary of $\mathcal{A} \times \mathcal{A}$, the curves $\Gamma_{\Delta}, \Gamma_{\text {char }}^{1, l}, \Gamma_{\text {char }}^{1, r}, \Gamma_{\text {char }}^{2, l}$, $\Gamma_{\text {char }}^{2, r}$, which are given for $k=1,2$ by

$$
\begin{aligned}
& \Gamma_{\text {char }}^{k, l}:=\left\{\left(\rho_{l}, \rho_{r}\right) \in \mathcal{A}^{2}, \rho_{l} \neq \rho_{r} \mid \exists\left(m_{l}, m_{r}, s\right) \in \mathbb{R}^{3}:(14),(22) \text { and } \lambda_{k}\left(\rho_{l}, m_{l}\right)=s\right\} \cup\left(\rho^{*}, \rho^{*}\right), \\
& \Gamma_{\text {char }}^{k, r}:=\left\{\left(\rho_{l}, \rho_{r}\right) \in \mathcal{A}^{2}, \rho_{l} \neq \rho_{r} \mid \exists\left(m_{l}, m_{r}, s\right) \in \mathbb{R}^{3}:(14),(22) \text { and } \lambda_{k}\left(\rho_{r}, m_{r}\right)=s\right\} \cup\left(\rho^{*}, \rho^{*}\right) .
\end{aligned}
$$

We note, that the conditions $\lambda_{k}\left(\rho_{l / r}, m_{l / r}\right)=s$ in (33) are equivalent to

$$
\sqrt{\tilde{p}_{\tau}\left(\tau_{l}\right)}=\sqrt{\frac{\tilde{p}\left(\tau_{r}\right)-\tilde{p}\left(\tau_{l}\right)}{\tau_{r}-\tau_{l}}} \text { or } \sqrt{\tilde{p}_{\tau}\left(\tau_{r}\right)}=\sqrt{\frac{\tilde{p}\left(\tau_{r}\right)-\tilde{p}\left(\tau_{l}\right)}{\tau_{r}-\tau_{l}}}
$$



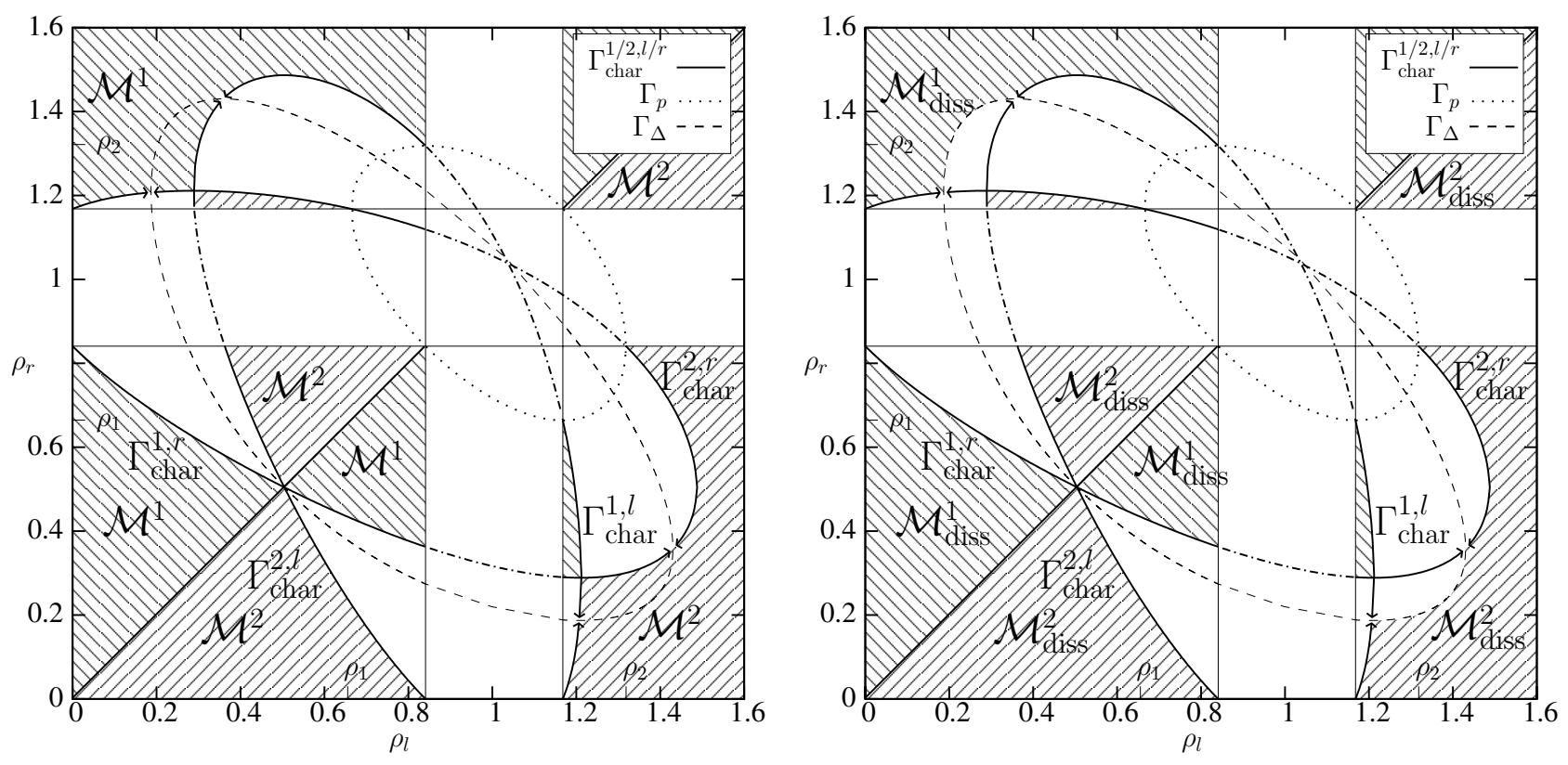

FIGURE 3. Regions of density values which can be connected by Laxian shock waves (left figure) and dissipative Laxian shock waves (right figure) are hatched. Note that Laxian waves can only be ruled out due to non-dissipativity if the states are in different phases. We recall that $\Gamma_{p}$ denotes the density pairs with identical pressure and $\Gamma_{\Delta}$ the pairs with zero entropy dissipation while point on the curves $\Gamma_{\text {char }}^{1 / 2, l / r}$ correspond to characteristic waves (cf. (33)). For $\rho_{1}$ and $\rho_{2}$ we refer to $(7)$.

They are in particular independent of momentum.

Assumption 1.1 ensures that there are exactly two specific densities $\tau_{7}$, $\tau_{8}$ with $0<\rho_{8}<\rho_{\min }^{\text {ellipt }}<\rho_{\max }^{\text {ellipt }}<\rho_{7}$ such that the slope of $\tilde{p}$ in both points and the slope of the straight line connecting $\left(\tau_{8}, \tilde{p}\left(\tau_{8}\right)\right)$ with $\left(\tau_{7}, \tilde{p}\left(\tau_{7}\right)\right)$ takes the same value (see Fig. 1). In view of the fact that intersection points of the curves defined in (33) have to satisfy both relations in $(34)$ we conclude that there is exactly one intersection point in $\left(0, \rho_{\min }^{\text {ellipt }}\right) \times\left(\rho_{\max }^{\text {ellipt }}, 1 / b\right)$ as well as one in $\left(\rho_{\max }^{\text {ellipt }}, 1 / b\right) \times\left(0, \rho_{\min }^{\text {ellipt }}\right)$, namely $(c f$. Fig. 4$)$

$$
\left(\rho_{8}, \rho_{7}\right) \text { and }\left(\rho_{7}, \rho_{8}\right) \text {. }
$$

In the next step we define the set

$$
\Gamma_{p}:=\left\{\left(\rho_{l}, \rho_{r}\right) \in \mathcal{A}^{2} \mid p\left(\rho_{l}\right)=p\left(\rho_{r}\right), \rho_{l} \neq \rho_{r}\right\}
$$

We observe that $\Gamma_{p}$ is symmetric with respect to the axis $\{(\rho, \rho) \mid \rho \in(0,1 / b)\}$.

Due to (34) and (7) the union of $\Gamma_{\text {char }}^{1, l}, \Gamma_{\text {char }}^{1, r}, \Gamma_{\text {char }}^{2, l}, \Gamma_{\text {char }}^{2, r}$ and the curve $\Gamma_{p}$ has four intersection points

$$
\left(\rho_{\min }^{\text {ellipt }}, \rho_{2}\right),\left(\rho_{2}, \rho_{\min }^{\text {ellipt }}\right),\left(\rho_{\max }^{\text {ellipt }}, \rho_{1}\right), \text { and }\left(\rho_{1}, \rho_{\max }^{\text {ellipt }}\right)
$$

The strict inequalities in (4) show that the points in (36) are the only ones. 
Recall from (24) that the entropy dissipation vanishes if and only if either $\rho_{l}=\rho_{r}, \Delta=0$ or $v_{l}=v_{r}=s$ hold. The latter case implies for states satisfying the Rankine-Hugoniot shock speed formula (19) that

$$
\llbracket p \rrbracket=0
$$

is valid.

Note, that due to (9) and (28) the curves $\Gamma_{\Delta}$ and $\Gamma_{p}$ intersect in the Maxwell points. These are the only intersection points, which can be seen easily from (26).

In the vapor phase the genuine nonlinearity breaks down exactly at states with $\rho=\rho^{*}$. As $\left(\rho^{*}, \rho^{*}\right) \in \Gamma_{\Delta}$ the curves from (33) and $\Gamma_{\Delta}$ intersect at the point $\left(\rho^{*}, \rho^{*}\right)$ in the vapor phase. This is the only intersection point in the vapor phase, which follows from the shape of $p$ (cf. Assumption 1.1) and (26).

There are exactly four further intersection points within $\mathcal{A}^{2}$ of $\Gamma_{\Delta}$ and the curves from (33) (see Fig. 4), namely

$$
\left(\rho_{3}, \rho_{4}\right), \quad\left(\rho_{4}, \rho_{3}\right), \quad\left(\rho_{5}, \rho_{6}\right), \quad \text { and } \quad\left(\rho_{6}, \rho_{5}\right) .
$$

For simplicity we only show that $\left(\rho_{4}, \rho_{3}\right) \in\left(\rho_{\max }^{\text {ellipt }}, 1 / b\right) \times\left(0, \rho_{\min }^{\text {ellipt }}\right)$ belongs to $\Gamma_{\Delta}$ while it is also an end point of $\Gamma_{\text {char }}^{1, r}$ and $\Gamma_{\text {char }}^{2, r}$ (see Fig. 4). Due to Assumption 1.1 there is a point $\hat{\tau} \in\left(b, 1 / \rho_{\max }^{\text {ellipt }}\right)$ such that

$$
\sqrt{\tilde{p}_{\tau}\left(\tau^{*}\right)}=\sqrt{\frac{\tilde{p}\left(\tau^{*}\right)-\tilde{p}(\hat{\tau})}{\tau^{*}-\hat{\tau}}}
$$

holds, i.e. $\left(\hat{\tau}, \tau^{*}\right)$ satisfies $(34)$ and due to $(25)$ the relation $\tilde{\Delta}\left(\hat{\tau}, \tau^{*}\right)<0$.

On the other hand, for $\left(\rho_{7}, \rho_{8}\right) \in \Gamma_{\text {char }}^{k, r}$ (see (35)) it holds $\tilde{\Delta}\left(1 / \rho_{7}, 1 / \rho_{8}\right)>0$. Therefore, there is an intersection point $\left(\rho_{4}, \rho_{3}\right) \in\left(\rho_{\max }^{\text {ellipt }}, 1 / b\right) \times\left(0, \rho_{\text {min }}^{\text {ellipt }}\right)$ of $\Gamma_{\Delta}$ and the curves from (33).

With help of monotonicity arguments and $\tilde{p}_{\tau \tau}>0$ in $\left(b, 1 / \rho_{\max }^{\text {ellipt }}\right)$ and $\tilde{p}_{\tau \tau}>0$ in $\left(\tau^{*}, 1 / \rho_{8}\right)$ one can show the uniqueness of this intersection point.

Remark 2.3 (Ordering and end points).

(i) For the intersection points identified above we have the ordering relations

$$
\begin{gathered}
\rho_{6}<\rho_{8}<\rho_{3}<\rho_{\min }^{\text {ellipt }}<\rho_{\max }^{\text {ellipt }}<\rho_{5}<\rho_{7}<\rho_{4}, \\
\rho_{1}<\rho_{\text {vapor }}^{M}<\rho_{\min }^{\text {ellipt }}<\rho_{\max }^{\text {ellipt }}<\rho_{\text {liquid }}^{M}<\rho_{2}, \\
\rho_{3}<\rho_{\text {vapor }}^{M}<\rho_{\min }^{\text {ellipt }}<\rho_{\max }^{\text {ellipt }}<\rho_{\text {liquid }}^{M}<\rho_{4} .
\end{gathered}
$$

The inequalities $\rho_{6}<\rho_{8}<\rho_{3}<\rho_{\min }^{\text {ellipt }}$ as well as $\rho_{\max }^{\text {ellipt }}<\rho_{5}<\rho_{7}<\rho_{4}$ can be verified using the definition of the curves $\Gamma_{\text {char }}^{1 / 2, l / r}$ and $\Gamma_{\Delta}(c f .(25))$ and Assumption 1.1.

$\rho_{1} \leq \rho_{\text {vapor }}^{M} \leq \rho_{\min }^{\text {ellipt }} \leq \rho_{\max }^{\text {ellipt }} \leq \rho_{\text {liquid }}^{M} \leq \rho_{2}$ follows directly from the definition of the points (see Fig. 1). The strict inequality " $<$ " then follows from $(26)$.

For $\rho_{4}>\rho_{3}$ it holds $p\left(\rho_{4}\right)>p\left(\rho_{3}\right)$ because $\left(\rho_{4}, \rho_{3}\right) \in \Gamma_{\text {char }}^{1 / 2, r}$ and therefore satisfies the Rankine-Hugoniot jump condition (14) for some $v_{l}, v_{r} \in \mathbb{R}$. Moreover, it holds $\Delta\left(\rho_{4}, \rho_{3}\right)=0$ as we have $\left(\rho_{4}, \rho_{3}\right) \in \Gamma_{\Delta}$ (cf. (38)). Now suppose $\rho_{3}>\rho_{\text {vapor }}^{M}\left(\rho_{4}<\rho_{\text {liquid }}^{M}\right.$ analogously). Then

$$
p\left(\rho_{4}\right)>p\left(\rho_{3}\right)>p\left(\rho_{\text {vapor }}^{M}\right)=p\left(\rho_{\text {liquid }}^{M}\right)
$$

and due to $(26) \Delta\left(\rho_{4}, \rho_{3}\right) \neq 0$. Therefore, the statement follows.

(ii) The endpoints of $\Gamma_{\text {char }}^{1 / 2, r}$ lying in $\mathcal{A}^{2}$ are exactly $\left(\rho_{4}, \rho_{3}\right)$ and $\left(\rho_{6}, \rho_{5}\right)$. Endpoints of $\Gamma_{\text {char }}^{1 / 2, l}$ lying in $\mathcal{A}^{2}$ are exactly $\left(\rho_{3}, \rho_{4}\right)$ and $\left(\rho_{5}, \rho_{6}\right)$. 


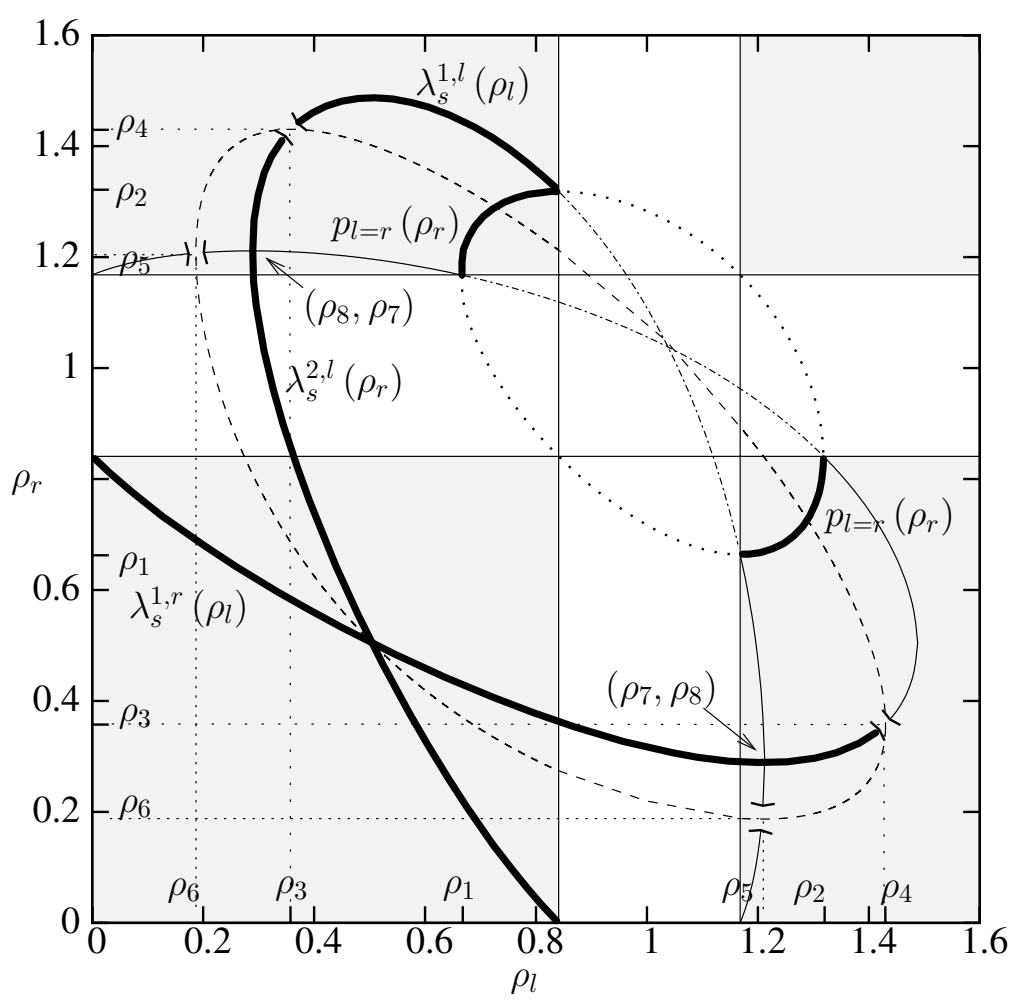

Figure 4. Graphs of the functions $\lambda_{s}^{1, l}, \lambda_{s}^{1, r}$ and $\lambda_{s}^{2, l}$ as defined in Lemma 2.4 and $p_{l=r}$ from Lemma 2.5. They consist of parts of the characteristic curves and the equal-pressure curve.

To prove this note that for all $\left(\rho_{l}, \rho_{r}\right)$ satisfying $(34)$ there exists $\left(m_{l}, m_{r}, s\right) \in \mathbb{R}^{3}$ such that (14) holds. Therefore, the only possible endpoints of $\Gamma_{\text {char }}^{1 / 2, r}$ within $\mathcal{A}^{2}$ are the points where the sign of the entropy dissipation changes, i.e. intersection points of $\Gamma_{\text {char }}^{1 / 2, r}$ and the set of points where the entropy dissipation (22) is zero. Due to (24) this can happen if $p\left(\rho_{l}\right)=p\left(\rho_{r}\right)\left(c f\right.$. (37)) or if $\Delta\left(\rho_{l}, \rho_{r}\right)=0$ holds. Intersection points of $\Gamma_{p}$ and $\Gamma_{\text {char }}^{1 / 2, r}$ are not in $\mathcal{A}^{2}$ (cf. (36)). Therefore, endpoints of $\Gamma_{\text {char }}^{1 / 2, r}$ within $\mathcal{A}^{2}$ have to be elements of $\Gamma_{\Delta}$. Due to (38) this is the case for $\left(\rho_{4}, \rho_{3}\right),\left(\rho_{6}, \rho_{5}\right)$.

The second part of the remark follows by symmetry.

With the above remarks we obtain the configuration as depicted in Figures 3 and 4 . The complexity of the two-phase case becomes visible if one recalls that the one-phase case for a perfect fluid leads to the simple situation in the liquid box $\left(\rho_{\max }^{\text {ellipt }}, 1 / b\right)^{2}$.

Parts of the sets $\Gamma_{\text {char }}^{1, l}, \Gamma_{\text {char }}^{1, r}, \Gamma_{\text {char }}^{2, l}$ and $\Gamma_{\text {char }}^{2, r}$ are graphs of functions depending on $\rho_{l}$ or $\rho_{r}$. We will define those functions by the subsequent lemma ( $c f$. also Fig. 4) as we need them in the remainder of the paper.

Lemma 2.4. There exist functions

$$
\begin{array}{ccccc}
\lambda_{s}^{1, r} & : & \left(0, \rho_{4}\right) & \longrightarrow & \left(0, \rho_{\min }^{\text {ellipt }}\right) \\
\lambda_{s}^{1, l} & : & \left(\rho_{3}, \rho_{\text {min }}^{\text {ellipt }}\right) & \longrightarrow & \left(\rho_{\text {max }}^{\text {ellipt }}, 1 / b\right) \\
\lambda_{s}^{2, l} & : & \left(0, \rho_{4}\right) & \longrightarrow & \left(0, \rho_{\min }^{\text {ellipt }}\right)
\end{array}
$$

such that

(i) $\Gamma_{\text {char }}^{1, r} \cap\left\{\left(\rho_{l}, \rho_{r}\right) \mid \rho_{r} \in\left(0, \rho_{\text {min }}^{\text {ellipt }}\right)\right\}=\left\{\left(\rho_{l}, \lambda_{s}^{1, r}\left(\rho_{l}\right)\right) \mid \rho_{l} \in\left(0, \rho_{4}\right)\right\}$, 
(ii) $\Gamma_{\text {char }}^{1, l} \cap\left\{\left(\rho_{l}, \rho_{r}\right) \mid \rho_{l} \in\left(0, \rho_{\text {min }}^{\text {ellipt }}\right)\right\}=\left\{\left(\rho_{l}, \lambda_{s}^{1, l}\left(\rho_{l}\right)\right) \mid \rho_{l} \in\left(\rho_{3}, \rho_{\text {min }}^{\text {ellipt }}\right)\right\}$,

(iii) $\Gamma_{\text {char }}^{2, l} \cap\left\{\left(\rho_{l}, \rho_{r}\right) \mid \rho_{l} \in\left(0, \rho_{\text {min }}^{\text {ellipt }}\right)\right\}=\left\{\left(\lambda_{s}^{2, l}\left(\rho_{r}\right), \rho_{r}\right) \mid \rho_{r} \in\left(0, \rho_{4}\right)\right\}$.

Proof. We prove (i) explicitly and note that (ii), (iii) can be done in the same manner. For $\rho_{l} \in\left(0, \rho_{4}\right)$ there are two solutions of the equation

$$
\tilde{p}_{\tau}(\tau)=\frac{\tilde{p}(\tau)-\tilde{p}\left(\tau_{l}\right)}{\tau-\tau_{l}}, \tau_{l}=\frac{1}{\rho_{l}}
$$

Define

$$
\lambda_{s}^{1, r}\left(\rho_{l}\right):=\min \left\{\frac{1}{\tau} \mid \tau \text { solves }(39)\right\} .
$$

Then, $\lambda_{s}^{1, r}\left(\rho_{l}\right) \in\left(0, \rho_{\text {min }}^{\text {ellipt }}\right)$, due to the shape of $p$ (cf. Assumption 1.1).

Moreover we get $\left(\rho_{l}, \lambda_{s}^{1, r}\left(\rho_{l}\right)\right) \in \Gamma_{\text {char }}^{1, r}$ as the condition $\lambda_{1}(\rho, m)=s$ is equivalent to (39) and the corresponding shocks are entropy dissipative $(22)$. We show the latter statement for a single point $\left(\rho_{l}, \lambda_{s}^{1, r}\left(\rho_{l}\right)\right)$ with $\rho_{l} \in$ $\left(0, \rho_{\text {min }}^{\text {ellipt }}\right)$. Then, the argument can be extended to $\rho_{l} \in\left(0, \rho_{4}\right)$ as the sign of the entropy dissipation does not change until the intersection point with $\Gamma_{\Delta}$, i.e. $\left(\rho_{4}, \rho_{3}\right)\left(c f\right.$. Rem. 2.3(ii)). Let for simplicity $\rho_{l} \in\left(\rho^{*}, \rho_{\text {min }}^{\text {ellipt }}\right)$. Due to $(24)$ we can calculate the sign of the entropy dissipation explicitly. As for $\left(\rho_{l}, \lambda_{s}^{1, r}\left(\rho_{l}\right)\right)$ the condition $\lambda_{1}\left(\rho_{l}, m_{l}\right)=s$ holds the corresponding shock wave can due to (29) only be a 1 -shock wave. Therefore, $j=$ $\rho_{l}\left(v_{l}-s\right)>0$ due to $(19)$ (with - sign for a 1-shock wave).

Moreover we have $\llbracket \rho \rrbracket^{3}>0$ as $\rho_{l}>\lambda_{s}^{1, r}\left(\rho_{l}\right)$ and $\Delta\left(\rho_{l}, \lambda_{s}^{1, r}\left(\rho_{l}\right)\right)>0$ due to (25).

Altogether, the entropy dissipation (24) is negative.

Accordingly, part of the set $\Gamma_{p}$ can be seen as the graph of a function depending on $\rho_{r}$.

Lemma 2.5. There is a function $p_{l=r}:\left(\rho_{1}, \rho_{\min }^{\text {ellipt }}\right) \cup\left(\rho_{\max }^{\text {ellipt }}, \rho_{2}\right) \longrightarrow\left(\rho_{1}, \rho_{\min }^{\text {ellipt }}\right) \cup\left(\rho_{\max }^{\text {ellipt }}, \rho_{2}\right)$ such that

$$
\Gamma_{p}=\left\{\left(p_{l=r}\left(\rho_{r}\right), \rho_{r}\right) \mid \rho_{r} \in\left(\rho_{1}, \rho_{\min }^{\text {ellipt }}\right) \cup\left(\rho_{\max }^{\text {ellipt }}, \rho_{2}\right)\right\}
$$

Proof. Without loss of generality let $\rho \in\left(\rho_{1}, \rho_{\min }^{\text {ellipt }}\right)$. Due to Assumption 1.1 there is exactly one $\hat{\rho} \in\left(\rho_{\max }^{\text {ellipt }}, \rho_{2}\right)$ such that $p(\hat{\rho})=p(\rho)$ holds. The statement follows with $p_{l=r}(\rho):=\hat{\rho}$.

Out of points on the Rankine-Hugoniot curves let us now select (the $\rho$-components) of those shock waves that satisfy the Lax entropy condition (29) and are entropy-dissipative.

Lemma 2.6 (1-shock waves). Let $\left(\rho_{l}, v_{l}\right)$ and $\left(\rho_{r}, v_{r}\right) \in \mathcal{A} \times \mathbb{R}$ be in one phase, such that (20) holds. Then (31) holds, if and only if we have

$$
\begin{array}{r}
\left(\rho_{l}, \rho_{r}\right) \in \mathcal{S}^{1}:=\left\{\left(\rho_{l}, \rho_{r}\right) \mid \rho_{l} \in\left(0, \rho^{*}\right), \rho_{r} \in\left(\rho_{l}, \lambda_{s}^{1, r}\left(\rho_{l}\right)\right]\right\} \cup\left\{\left(\rho_{l}, \rho_{r}\right) \mid \rho_{l} \in\left(\rho^{*}, \rho_{\text {min }}^{\text {ellipt }}\right), \rho_{r} \in\left[\lambda_{s}^{1, r}\left(\rho_{l}\right), \rho_{l}\right)\right\} \\
\cup\left\{\left(\rho_{l}, \rho_{r}\right) \mid \rho_{l} \in\left(\rho_{\text {max }}^{\text {ellipt }}, 1 / b\right), \rho_{r} \in\left(\rho_{l}, 1 / b\right)\right\} .
\end{array}
$$

Moreover we have $\left(\rho_{l}, \rho_{r}\right) \in \mathcal{M}_{\mathrm{diss}}^{1}$, thus the waves are entropy-dissipative.

Proof. We show the statement for $\rho_{l} \in\left(0, \rho^{*}\right)$. The other cases are proved similarly. Let $\left(\rho_{l}, m_{l}\right)$ and $\left(\rho_{r}, m_{r}\right) \in$ $\mathcal{A} \times \mathbb{R}$ be in one phase, such that (20) holds. We have to verify (31) for $\rho_{r} \in\left(\rho_{l}, \lambda_{s}^{1, r}\left(\rho_{l}\right)\right)$. Therefore, first assume $\rho_{l}>\rho_{r}$, i.e. $\tau_{l}<\tau_{r}$. Then as $\tau_{l}>\tau^{*}$ and $\tilde{p}_{\tau \tau}(\tau)>0$ for $\tau>\tau^{*}$ the following relation holds (cf. Fig. 5a):

$$
\tilde{p}_{\tau}\left(\tau_{l}\right)<\frac{\tilde{p}\left(\tau_{r}\right)-\tilde{p}\left(\tau_{l}\right)}{\tau_{r}-\tau_{l}}<\tilde{p}_{\tau}\left(\tau_{r}\right)
$$

and therefore, (31) does not hold. Hence, equation (29) does not hold for $\rho_{l}>\rho_{r}$. 


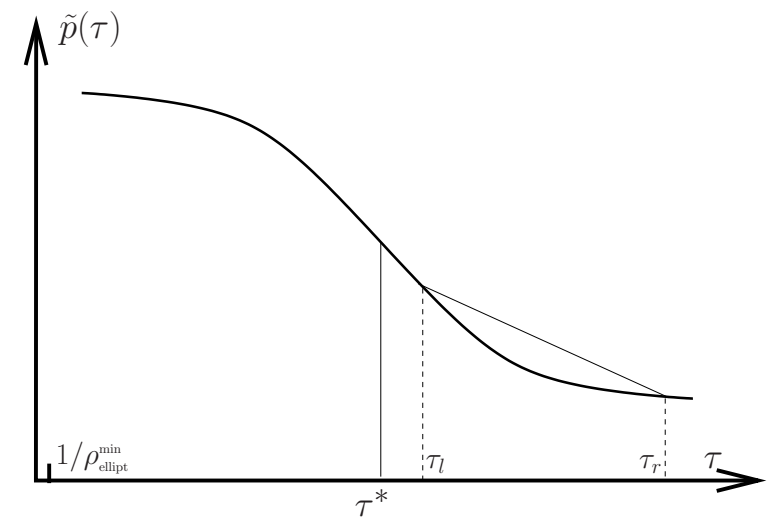

(a)

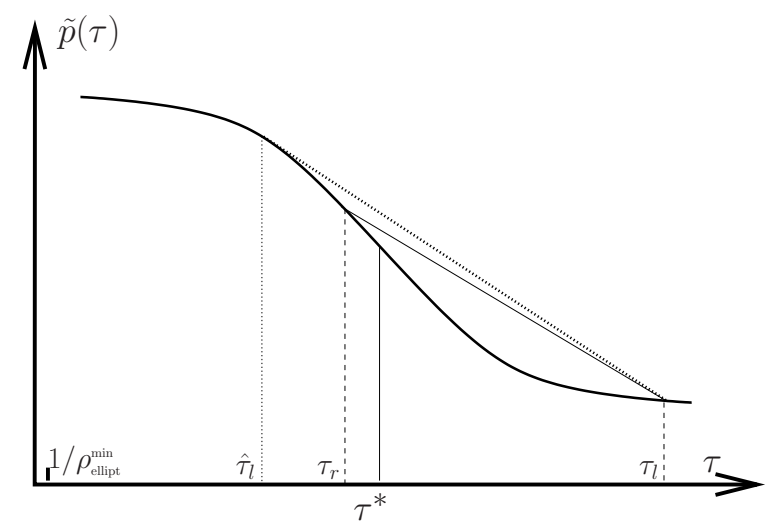

(b)

Figure 5. Auxiliary illustration for the proof of Lemma 2.6.

Now assume $\rho_{r}>\rho_{l}$, i.e. $\tau_{l}>\tau_{r}$, then as $\tau_{l}>\tau^{*}(31)$ holds, as long as $\tau_{r}>\hat{\tau}_{l}\left(i . e . \rho_{r}<\hat{\rho}_{l}:=\frac{1}{\hat{\tau}_{l}}\right)$, where $\hat{\tau}_{l}$ is defined as the unique solution of (see Fig. 5b)

$$
\frac{\tilde{p}\left(\hat{\tau}_{l}\right)-\tilde{p}\left(\tau_{l}\right)}{\hat{\tau}_{l}-\tau_{l}}=\tilde{p}_{\tau}\left(\hat{\tau}_{l}\right) .
$$

The uniqueness follows from the shape of the function $p$.

Now it remains to prove $\hat{\rho}_{l}=\lambda_{s}^{1, r}\left(\rho_{l}\right)$. This is true due to the definition of the function $\lambda_{s}^{1, r}$. Therefore, (29) is valid for $\rho_{r} \in\left(\rho_{l}, \lambda_{s}^{1, r}\left(\rho_{l}\right)\right)$. The cases $\rho_{l} \in\left(\rho^{*}, \rho_{\min }^{\text {ellipt }}\right)$ and $\rho_{l} \in\left(\rho_{\max }^{\text {ellipt }}, 1 / b\right)$ are proved similarly. Finally, we observe from the location of $\mathcal{M}_{\text {diss }}^{1}$ the inclusion $\mathcal{S}^{1} \subset \mathcal{M}_{\text {diss }}^{1}$ which concludes the proof.

Equivalently for 2-shock waves the following lemma is valid:

Lemma 2.7 (2-shock waves). Let $\left(\rho_{l}, v_{l}\right)$ and $\left(\rho_{r}, v_{r}\right) \in \mathcal{A} \times \mathbb{R}$ be in one phase, such that (21) holds. Then (32) holds, iff

$$
\begin{array}{r}
\left(\rho_{l}, \rho_{r}\right) \in \mathcal{S}^{2}:=\left\{\left(\rho_{l}, \rho_{r}\right) \mid \rho_{r} \in\left(0, \rho^{*}\right), \rho_{l} \in\left(\rho_{r}, \lambda_{s}^{2, l}\left(\rho_{r}\right)\right)\right\} \cup\left\{\left(\rho_{l}, \rho_{r}\right) \mid \rho_{r} \in\left(\rho^{*}, \rho_{\text {min }}^{\text {ellipt }}\right), \rho_{l} \in\left(\lambda_{s}^{2, l}\left(\rho_{r}\right), \rho_{r}\right)\right\} \\
\cup\left\{\left(\rho_{l}, \rho_{r}\right) \mid \rho_{r} \in\left(\rho_{\max }^{\text {ellipt }}, 1 / b\right), \rho_{l} \in\left(\rho_{r}, 1 / b\right)\right\}
\end{array}
$$

Moreover we have $\left(\rho_{l}, \rho_{r}\right) \in \mathcal{M}_{\mathrm{diss}}^{2}$.

The sets $\mathcal{S}^{1}$ and $\mathcal{S}^{2}$ are shown in Figures 6 and 7.

\subsection{Rarefaction waves and attached waves}

Next let us review the structure of rarefaction waves. Let $\left(\rho_{l}, m_{l}\right),\left(\rho_{r}, m_{r}\right) \in \mathcal{A} \times \mathbb{R}$ with $\rho_{l}$ and $\rho_{r}$ from the same phase be given. For $k \in\{1,2\}$ a $k$-rarefaction wave (denoted by $\left.\left(\rho_{l}, m_{l}\right) \stackrel{\text { rare }}{\rightarrow}\left(\rho_{r}, m_{r}\right)\right)$ is a weak solution $(\rho, m)^{T}: \mathbb{R} \times[0, \infty) \rightarrow \mathcal{A} \times \mathbb{R}$ of $(1)$ of the type

$$
\left(\begin{array}{c}
\rho(x, t) \\
m(x, t)
\end{array}\right)=\left\{\begin{array}{cl}
\left(\begin{array}{c}
\rho_{l} \\
m_{l}
\end{array}\right) & : \quad x \leq \xi_{1}^{k} t \\
\left(\begin{array}{c}
\bar{\rho}(x / t) \\
\bar{m}(x / t)
\end{array}\right) & : \xi_{1}^{k} t<x<\xi_{2}^{k} t \\
\left(\begin{array}{c}
\rho_{r} \\
m_{r}
\end{array}\right) & : \quad x \geq \xi_{2}^{k} t
\end{array}\right.
$$




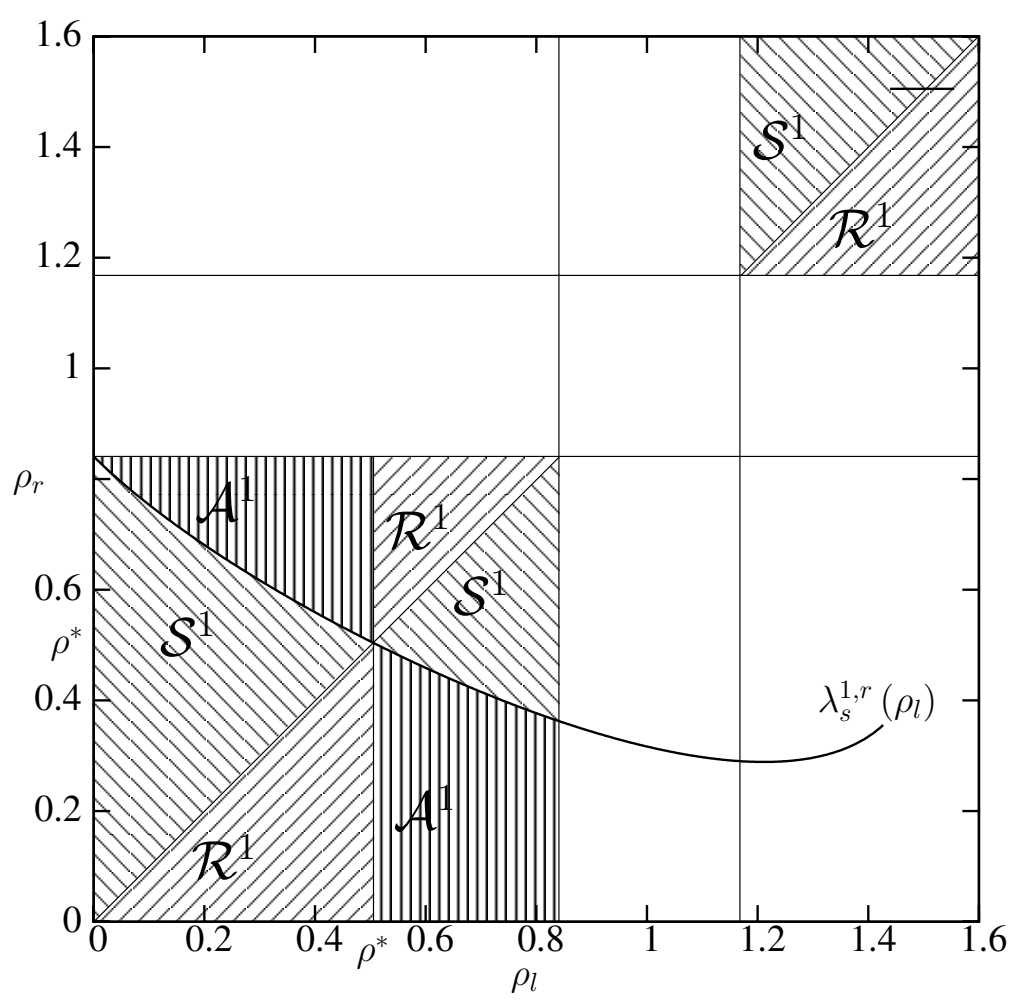

Figure 6. Classical 1-waves: density components of states $\left(\rho_{l}, v_{l}\right)$ and $\left(\rho_{r}, v_{r}\right)$ within one phase can be connected by a classical 1 -wave. $\mathcal{S}^{1}$ corresponds to an 1 -shock wave $\left(c f\right.$. Lem. 2.6), $\mathcal{R}^{1}$ to a 1-rarefaction wave ( $c f$. Lem. 2.9) and $\mathcal{A}^{1}$ to an attached 1-wave ( $c f$. Lem. 2.10).

Here $(\bar{\rho}, \bar{m})^{T} \in C^{1}\left(\left(\xi_{1}^{i}, \xi_{2}^{i}\right), \mathcal{A} \times \mathbb{R}\right)$ has to be chosen such that $(\rho, m)^{T}$ is a classical solution of $(1)$ for $\xi_{1}^{k} t<$ $x<\xi_{2}^{k} t$ with the property

$$
\bar{\rho}\left(\xi_{1}^{k}\right)=\rho_{l}, \bar{\rho}\left(\xi_{2}^{k}\right)=\rho_{r}, \bar{m}\left(\xi_{1}^{k}\right)=\bar{\rho} \bar{v}\left(\xi_{1}^{k}\right)=m_{l}, \bar{m}\left(\xi_{2}^{k}\right)=\bar{\rho} \bar{v}\left(\xi_{2}^{k}\right)=m_{r},
$$

where we have

$$
\xi_{1}^{k}=\lambda_{k}\left(\rho_{l}, m_{l}\right), \xi_{2}^{k}=\lambda_{k}\left(\rho_{r}, m_{r}\right) .
$$

For the hydrodynamical equations (1) the functions $\bar{\rho}$ and $\bar{m}=\bar{\rho} \bar{v}$ have to satisfy (41) and for $\xi \in\left(\xi_{1}^{k}, \xi_{2}^{k}\right)$ have to be real solutions of the system of ordinary differential equations

$$
\begin{aligned}
\bar{\rho}_{1 / 2}^{\prime}(\xi) & =\mp \frac{2 \bar{\rho}(\xi) \sqrt{p^{\prime}(\bar{\rho}(\xi))}}{\bar{\rho}(\xi) p^{\prime \prime}(\bar{\rho}(\xi))+2 p^{\prime}(\bar{\rho}(\xi))}, \\
(\bar{\rho} \bar{v})_{1 / 2}^{\prime}(\xi) & =\bar{\rho}_{1 / 2}^{\prime}(\xi)\left(\bar{v}(\xi) \mp \sqrt{p^{\prime}(\bar{\rho}(\xi))}\right) .
\end{aligned}
$$

Eliminating $\xi$ in the above equations we can express $\bar{v}$ in terms of $\bar{\rho}$ :

$$
\bar{v}(\bar{\rho})=v_{l} \mp \int_{\rho_{l}}^{\bar{\rho}} \frac{\sqrt{p^{\prime}(r)}}{r} \mathrm{~d} r .
$$

With (43) we have got a parameterization in the $(\rho, m)$-plane of the rarefaction curves, i.e. the set of states $\left(\rho_{r}, m_{r}\right) \in \mathcal{A} \times \mathbb{R}$ that can be connected by a $k$-rarefaction wave to $\left(\rho_{l}, m_{l}\right)$. 


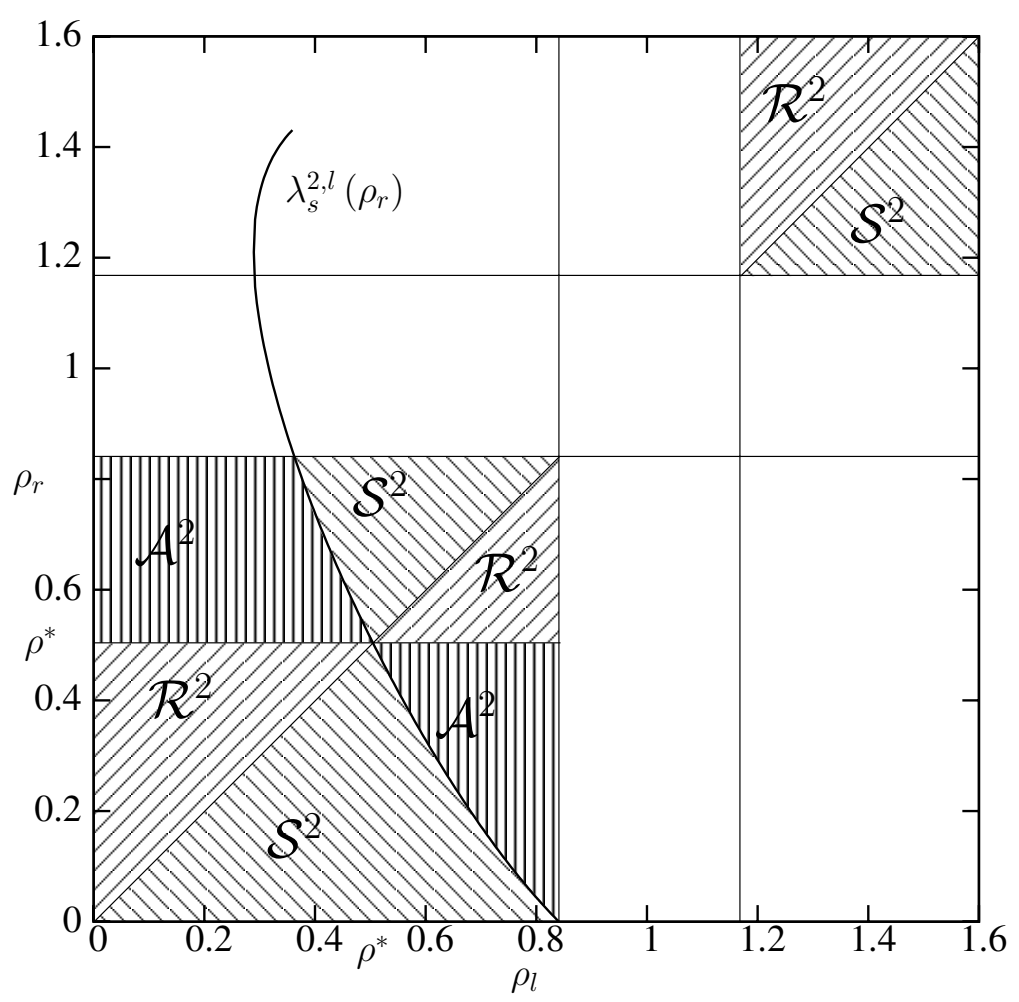

Figure 7. Classical 2-waves: density components of states $\left(\rho_{l}, v_{l}\right)$ and $\left(\rho_{r}, v_{r}\right)$ within one phase can be connected by a classical 2 -wave. $\mathcal{S}^{2}$ corresponds to a 2 -shock wave ( $f$. Lem. 2.7), $\mathcal{R}^{2}$ to a 2-rarefaction wave ( $c f$. Lem. 2.9) and $\mathcal{A}^{2}$ to an attached wave ( $c f$. Lem. 2.10).

Remark 2.8. The parameterizations of the rarefaction curves given in (42) with $v_{r}$ as a function of $\rho_{r}$ have the monotonicity property

$$
\frac{\mathrm{d} v_{r}}{\mathrm{~d} \rho_{r}}\left(\rho_{r}\right)<0(>0) \quad \text { for a 1-rarefaction wave (2-rarefaction wave) }
$$

This can be seen from

$$
\frac{\mathrm{d} v_{r}}{\mathrm{~d} \rho_{r}}\left(\rho_{r}\right)=\mp \frac{\sqrt{p^{\prime}\left(\rho_{r}\right)}}{\rho_{r}},
$$

with "-" for a 1-rarefaction wave and "+" for a 2-rarefaction wave.

It is well-known (see [11]) that the system of ordinary differential equations (42) has a unique solution if the condition (cf. (12))

$$
\Lambda_{\mp}=\nabla \lambda_{k} \cdot \vec{r}_{i}>0, \quad k \in\{1,2\}
$$


is satisfied along the wave. This leads to:

Lemma 2.9 (1/2-rarefaction waves). Let $\left(\rho_{l}, m_{l}\right)$ and $\left(\rho_{r}, m_{r}\right) \in \mathcal{A} \times \mathbb{R}$ be within one phase such that (43) holds. Then (44) holds, if and only if

$$
\begin{array}{r}
\left(\rho_{l}, \rho_{r}\right) \in \mathcal{R}^{1}:=\left\{\left(\rho_{l}, \rho_{r}\right) \mid \rho_{l} \in\left(0, \rho^{*}\right), \rho_{r} \in\left(0, \rho_{l}\right)\right\} \cup\left\{\left(\rho_{l}, \rho_{r}\right) \mid \rho_{l} \in\left(\rho^{*}, \rho_{\text {min }}^{\text {ellipt }}\right), \rho_{r} \in\left(\rho_{l}, \rho_{\text {min }}^{\text {ellipt }}\right)\right\} \\
\cup\left\{\left(\rho_{l}, \rho_{r}\right) \mid \rho_{l} \in\left(\rho_{\text {max }}^{\text {ellipt }}, 1 / b\right), \rho_{r} \in\left(\rho_{\text {max }}^{\text {ellipt }}, \rho_{l}\right)\right\}
\end{array}
$$

for a 1-rarefaction wave and

$$
\begin{array}{r}
\left(\rho_{l}, \rho_{r}\right) \in \mathcal{R}^{2}:=\left\{\left(\rho_{l}, \rho_{r}\right) \mid \rho_{r} \in\left(0, \rho^{*}\right), \rho_{l} \in\left(0, \rho_{r}\right)\right\} \cup\left\{\left(\rho_{l}, \rho_{r}\right) \mid \rho_{r} \in\left(\rho^{*}, \rho_{\text {min }}^{\text {ellipt }}\right), \rho_{l} \in\left(\rho_{r}, \rho_{\text {min }}^{\text {ellipt }}\right)\right\} \\
\cup\left\{\left(\rho_{l}, \rho_{r}\right) \mid \rho_{r} \in\left(\rho_{\text {max }}^{\text {ellipt }}, 1 / b\right), \rho_{l} \in\left(\rho_{\text {max }}^{\text {ellipt }}, \rho_{r}\right)\right\}
\end{array}
$$

for a 2-rarefaction wave.

Proof. Note that for a 1-rarefaction wave (cf. (12))

$$
\Lambda_{\mp}(\tau)>0 \Longleftrightarrow \tilde{p}^{\prime \prime}(\tau)<0 \Longleftrightarrow \rho \in\left(\rho^{*}, \rho^{* *}\right)
$$

holds. Now let $\left(\rho_{l}, v_{l}\right)$ and $\left(\rho_{r}, v_{r}\right)$ be two states on the rarefaction wave (43) (with negative sign for a 1 rarefaction wave).

If $\rho_{l} \in\left(\rho^{*}, \rho_{\text {min }}^{\text {ellipt }}\right) \subset\left(\rho^{*}, \rho^{* *}\right)$ holds then we have $\lambda_{1}\left(\rho_{r}, m_{r}\right)>\lambda_{1}\left(\rho_{l}, m_{l}\right)$ for $\rho_{r} \in\left(\rho_{l}, \rho_{\text {min }}^{\text {ellipt }}\right)$ since $\nabla \lambda_{1} \cdot \vec{r}_{1}>0$ on the wave.

If $\rho_{l} \in\left(0, \rho^{*}\right) \cup\left(\rho^{* *}, 1 / b\right)$ holds then we have $\lambda_{1}\left(\rho_{r}, m_{r}\right)>\lambda_{1}\left(\rho_{l}, m_{l}\right)$ for $\rho_{r}<\rho_{l}$.

The statement for 2-rarefaction waves is proved analogously.

The sets $\mathcal{R}^{1}$ and $\mathcal{R}^{2}$ are shown in Figures 6 and 7 . Note that we have no difficulties to satisfy the ClausiusDuhem inequality (22) for rarefaction waves as continuous weak solutions.

We observe from Figure 6 that we cannot connect all states in a single phase by only Laxian shock waves and rarefaction waves. Furthermore, we need attached waves.

Let $\left(\rho_{l}, m_{l}\right),\left(\rho_{r}, m_{r}\right) \in \mathcal{A} \times \mathbb{R}$. If there exists a state $(\hat{\rho}, \hat{m})$ such that an 1 -shock wave $\left(\rho_{l}, m_{l}\right) \stackrel{s}{\rightarrow}(\hat{\rho}, \hat{m})$ with speed $s=\lambda_{1}(\hat{\rho}, \hat{m})$ and an 1-rarefaction wave $(\hat{\rho}, \hat{m}) \stackrel{\text { rare }}{\rightarrow}\left(\rho_{r}, m_{r}\right)$ exist we say that $\left(\rho_{l}, m_{l}\right)$ and $\left(\rho_{r}, m_{r}\right)$ can be connected by an 1-attached wave (with notation $\left(\rho_{l}, m_{l}\right) \stackrel{\text { att }}{\rightarrow}\left(\rho_{r}, m_{r}\right)$. Obviously, the function

$$
\left(\begin{array}{c}
\rho(x, t) \\
m(x, t)
\end{array}\right)=\left\{\begin{array}{cl}
\left(\begin{array}{c}
\rho_{l} \\
m_{l}
\end{array}\right) & : \quad x<\lambda_{1}(\hat{\rho}, \hat{m}) t \\
\left(\begin{array}{c}
\bar{\rho}(x / t) \\
\bar{m}(x / t)
\end{array}\right) & : \quad \lambda_{1}(\hat{\rho}, \hat{m}) t \leq x<\lambda_{1}\left(\rho_{r}, m_{r}\right) t \\
\left(\begin{array}{c}
\rho_{r} \\
m_{r}
\end{array}\right) & : \quad x \geq \lambda_{1}\left(\rho_{r}, m_{r}\right) t
\end{array}\right.
$$

is then a weak solution of $(1)$, where $\bar{\rho}, \bar{m}$ are defined analogously as in (40) but with $(\hat{\rho}, \hat{m})$ as lefthand state. Similarly, if there exists a state $(\hat{\rho}, \hat{m})$ such that a 2-rarefaction wave $\left(\rho_{l}, m_{l}\right) \stackrel{\text { rare }}{\rightarrow}(\hat{\rho}, \hat{m})$ and a 2-shock wave $(\hat{\rho}, \hat{m}) \stackrel{s}{\rightarrow}\left(\rho_{r}, m_{r}\right)$ with speed $s=\lambda_{2}(\hat{\rho}, \hat{m})$ exist we say that $\left(\rho_{l}, m_{l}\right)$ and $\left(\rho_{r}, m_{r}\right)$ can be connected by an 2 -attached wave. Then, the function

$$
\left(\begin{array}{c}
\rho(x, t) \\
m(x, t)
\end{array}\right)=\left\{\begin{array}{cl}
\left(\begin{array}{c}
\rho_{l} \\
m_{l}
\end{array}\right) & : \quad x<\lambda_{2}\left(\rho_{l}, m_{l}\right) t \\
\left(\begin{array}{c}
\bar{\rho}(x / t) \\
\bar{m}(x / t)
\end{array}\right) & : \quad \lambda_{2}\left(\rho_{l}, m_{l}\right) t \leq x<\lambda_{2}(\hat{\rho}, \hat{m}) t \\
\left(\begin{array}{c}
\rho_{r} \\
m_{r}
\end{array}\right) & : \quad x \geq \lambda_{2}(\hat{\rho}, \hat{m}) t
\end{array}\right.
$$

is a weak solution of (1), where $\bar{\rho}, \bar{m}$ are defined analogously as in (40) but with $(\hat{\rho}, \hat{m})$ as righthand state.

The attached waves within one phase are described in the following lemma. 
Lemma $2.10\left(1 / 2\right.$-attached waves). Let $\left(\rho_{l}, v_{l}\right),\left(\rho_{r}, v_{r}\right) \in \mathcal{A} \times \mathbb{R}$ with densities in the vapor phase. They can be connected by an 1-attached wave $\left(\rho_{l}, m_{l}\right) \stackrel{\text { att }}{\rightarrow}\left(\rho_{r}, m_{r}\right)$, if and only if we have (cf. Fig. 6)

$$
\left(\rho_{l}, \rho_{r}\right) \in \mathcal{A}^{1}:=\left\{\left(\rho_{l}, \rho_{r}\right) \mid \rho_{l} \in\left(0, \rho^{*}\right), \rho_{r} \in\left(\lambda_{s}^{1, r}\left(\rho_{l}\right), \rho_{\min }^{\text {ellipt }}\right)\right\} \cup\left\{\left(\rho_{l}, \rho_{r}\right) \mid \rho_{l} \in\left(\rho^{*}, \rho_{\min }^{\text {ellipt }}\right), \rho_{r} \in\left(0, \lambda_{s}^{1, r}\left(\rho_{l}\right)\right)\right\}
$$

and by a 2-attached wave $\left(\rho_{l}, m_{l}\right) \stackrel{\text { att }}{\rightarrow}\left(\rho_{r}, m_{r}\right)$, if and only if we have (cf. Fig. 7$)$

$$
\left(\rho_{l}, \rho_{r}\right) \in \mathcal{A}^{2}:=\left\{\left(\rho_{l}, \rho_{r}\right) \mid \rho_{r} \in\left(0, \rho^{*}\right), \rho_{l} \in\left(\lambda_{s}^{2, l}\left(\rho_{r}\right), \rho_{\min }^{\text {ellipt }}\right)\right\} \cup\left\{\left(\rho_{l}, \rho_{r}\right) \mid \rho_{r} \in\left(\rho^{*}, \rho_{\text {min }}^{\text {ellipt }}\right), \rho_{l} \in\left(0, \lambda_{s}^{2, l}\left(\rho_{r}\right)\right)\right\}
$$

In both cases the Clausius-Duhem inequality (22) is satisfied.

Proof. The proof is done for the case of a 1-attached wave and for simplicity we only consider the case of $\rho_{l} \in\left(0, \rho^{*}\right)$. Values of $\rho_{r} \in\left(0, \lambda_{s}^{1, r}\left(\rho_{l}\right)\right)$ can only be reached by a single 1-rarefaction or one-shock wave. Bigger values of $\rho_{r}$ cannot be connected to $\rho_{l}$ by a single shock or rarefaction wave (Lems. 2.6 and 2.9).

The only way to connect $\left(\rho_{l}, m_{l}\right)$ to $\left(\rho_{r}, m_{r}\right)$ is therefore by a characteristic 1 -shock wave $\left(\rho_{l}, m_{l}\right) \stackrel{s}{\rightarrow}(\hat{\rho}:=$ $\left.\lambda_{s}^{1, r}\left(\rho_{l}\right), \hat{m}\right)$ with speed $s$ followed by an one-rarefaction wave $(\hat{\rho}, \hat{m}) \stackrel{\text { rare }}{\rightarrow}\left(\rho_{r}, m_{r}\right)$. For this construction $s=\lambda_{1}(\hat{\rho}, \hat{m})$ holds which follows from the definition of $\lambda_{s}^{1, r}$. This defines also uniquely the intermediate momentum $\hat{m}$ by the Rankine-Hugoniot conditions. The location of the set $\mathcal{M}_{\text {diss }}^{1}$ shows that the characteristic shock wave is entropy dissipative and thus (22) is satisfied for the whole attached curve.

The corresponding sets $\mathcal{A}^{1}$ and $\mathcal{A}^{2}$ are shown in Figures 6 and 7 .

Remark 2.11. With the consecutive use of (20), (21) and (43) there is a parameterization of the $k$-attached waves, $k \in\{1,2\}$, in the $(\rho, v)$-plane and due to the monotonicity properties of the parameterizations of the $k$-shock and $k$-rarefaction curves (see Rems. 2.2 and 2.8) it holds that $v_{r}=v_{r}\left(\rho_{r}\right)$ is monotone decreasing for a 1-attached wave and monotone increasing for a 2 -attached wave.

\subsection{Kinetic relation and subsonic phase transitions}

Up to now we have clarified the possible wave structure in one phase. To take into account phase transition let us note that the solution of the Riemann problem containing undercompressive shock waves which turns out to be necessary is - in contrast to the classical hyperbolic case - not unique any more [23]. One remedy of this problem is to admit only those undercompressive shock waves that satisfy an additional single algebraic constraint, the so-called kinetic relation. Note that undercompressive shock waves are automatically phase transitions in our case. The kinetic relation was first proposed by [1] (see also [39,40]) and motivated by experiments in the case of nonlinear elasticity. For the system (1) mathematically a similar situation occurs [6,12] so that it suggests itself to carry over this concept.

In the following we work with a specific kinetic relation.

Definition 2.12 (Kinetic relation). Let $\rho_{k r} \in\left(\rho_{\text {vapor }}^{M}, \rho_{4}\right)$.

The function $\Psi:\left(\lambda_{s}^{1, r}\left(\rho_{k r}\right), \rho_{\min }^{\text {ellipt }}\right) \cup\left(\rho_{\max }^{\text {ellipt }}, \lambda_{s}^{1, l}\left(\rho^{*}\right)\right) \longrightarrow\left(\rho^{*}, \rho_{\text {min }}^{\text {ellipt }}\right) \cup\left(\rho_{\text {max }}^{\text {ellipt }}, \rho_{k r}\right)$ given by

$$
\Psi(\rho):=\left\{\begin{array}{cl}
\rho_{\text {liquid }}^{M}+\frac{\rho_{k r}-\rho_{\text {liquid }}^{M}}{\lambda_{s}^{1, r}\left(\rho_{k r}\right)-\rho_{\text {vapor }}^{M}}\left(\rho-\rho_{\text {vapor }}^{M}\right) & : \quad \lambda_{s}^{1, r}\left(\rho_{k r}\right) \leq \rho<\rho_{\text {vapor }}^{M}, \\
p_{l=r}(\rho) & : \quad \rho_{\text {vapor }}^{M} \leq \rho<\rho_{\text {min }}^{\text {ellipt }}, \\
p_{l=r}(\rho) & : \quad \rho_{\text {max }}^{\text {ellipt }}<\rho \leq \rho_{\text {liquid }}^{M}, \\
\rho^{*}+\frac{\rho_{\text {vapor }}^{M}-\rho^{*}}{\rho_{\text {liquid }}^{M}-\lambda_{s}^{1, l}\left(\rho^{*}\right)}\left(\rho-\lambda_{s}^{1, l}\left(\rho^{*}\right)\right) & : \quad \rho_{\text {liquid }}^{M}<\rho \leq \lambda_{s}^{1, l}\left(\rho^{*}\right)
\end{array}\right.
$$

is called kinetic relation.

A phase transition $\left(\rho_{l}, m_{l}\right) \stackrel{p t}{\rightarrow}\left(\rho_{r}, m_{r}\right)$ is called $\Psi$-admissible if and only if $\rho_{l}=\Psi\left(\rho_{r}\right)$ holds. 
As can be checked easily using $\rho_{4}>\rho_{\text {liquid }}^{M}$ and $\rho_{3}<\rho_{\text {vapor }}^{M}$ (see Rem. 2.3) the kinetic relation from Definition 2.12 satisfies

$$
\frac{\partial}{\partial \rho_{r}} \Psi\left(\rho_{r}\right)<0 \text { for } \rho_{r} \in\left(\lambda_{s}^{1, r}\left(\rho_{k r}\right), \rho_{\text {vapor }}^{M}\right) \cup\left(\rho_{\text {liquid }}^{M}, \lambda_{s}^{1, l}\left(\rho^{*}\right)\right)
$$

In fact (48) is the crucial property to construct solutions for the Riemann problem (see Lem. 3.1) and we can formulate similar existence theorems for more general kinetic relations satisfying (48).

\section{Remark 2.13.}

(i) Up to our knowledge explicit formulae for kinetic relations in the case of liquid vapor have not been suggested. In this sense Definition 2.12 appears to be quite arbitrary. However, note that the Maxwell states $\rho_{\text {vapor }}^{M}$ and $\rho_{\text {liquid }}^{M}$ are connected by the kinetic relation so that the equilibrium configuration connecting the Maxwell states is $\Psi$-admissible. Then we use Laxian shocks whenever possible which implies that the kinetic relation is chosen to end in the characteristic curves and is not extended further. As the most simple choice we then take a linear function.

(ii) Mathematically one has to study whether the solution of the Riemann problem depends continuously on the choice of the kinetic function. Our conjecture is that the qualitative structure of the solution of the Riemann problem does not depend sensitively on the kinetic function (see [27], Sect. 2.3.1.8) for Riemann solutions to different kinetic relations). A general theory on continuous dependence with respect to the kinetic relation can be found in [9]. It is out of the scope of this work to apply the theory in [9] to our case but it is likely that continuous dependence can be proven rigorously.

(iii) A $\Psi$-admissible phase transition $\left(\rho_{l}, m_{l}\right) \stackrel{p t}{\rightarrow}\left(\rho_{r}, m_{r}\right)$ is entropy-dissipative: it is clear that it satisfies the inequality if one of the density states is given by the function $p_{l=r}$ since these jumps have zero entropy dissipation. In the other cases it follows from the location of the curve $\Gamma_{\Delta}$ with respect to the connecting states (see Fig. 8).

\section{Generalized wave curves and solution of the Riemann problem}

\subsection{Generalized 1-wave curve}

Before we can state the main theorem - the construction of a generalized 1-wave curve - we need to define an additional function $g$ which we will use in its proof. With the help of this function we make sure that the speeds of the elementary waves within the generalized 1-wave curve are such that they do not interact.

Lemma 3.1. There exists a function $g:\left(0, \rho_{\text {min }}^{\text {ellipt }}\right) \longrightarrow\left(\rho_{\text {liquid }}^{M}, \lambda_{s}^{1, l}\left(\rho^{*}\right)\right)$, such that for $\rho_{l} \in\left(0, \rho_{\text {min }}^{\text {ellipt }}\right)$

$$
\frac{\tilde{p}\left(g\left(\rho_{l}\right)^{-1}\right)-\tilde{p}\left(\Psi\left(g\left(\rho_{l}\right)\right)^{-1}\right)}{g\left(\rho_{l}\right)^{-1}-\Psi\left(g\left(\rho_{l}\right)\right)^{-1}}=\frac{\tilde{p}\left(\rho_{l}^{-1}\right)-\tilde{p}\left(\Psi\left(g\left(\rho_{l}\right)\right)^{-1}\right)}{\rho_{l}^{-1}-\Psi\left(g\left(\rho_{l}\right)\right)^{-1}} .
$$

Proof. Let us first define the functions $h_{1}, h_{2, l}:\left[1 / \lambda_{s}^{1, l}\left(\rho^{*}\right), 1 / \rho_{\text {liquid }}^{M}\right] \rightarrow \mathbb{R}$ as

$$
h_{1}(\tau):=\frac{\tilde{p}(\tau)-\tilde{p}(\tilde{\Psi}(\tau))}{\tau-\tilde{\Psi}(\tau)} \quad \text { and } \quad h_{2, l}(\tau):=\frac{\tilde{p}\left(\tau_{l}\right)-\tilde{p}(\tilde{\Psi}(\tau))}{\tau_{l}-\tilde{\Psi}(\tau)}
$$

where $\tilde{\Psi}(\tau):=1 / \Psi(1 / \tau)$. Then, it holds

$$
h_{1}\left(1 / \lambda_{s}^{1, l}\left(\rho^{*}\right)\right)=\frac{\tilde{p}\left(1 / \lambda_{s}^{1, l}\left(\rho^{*}\right)\right)-\tilde{p}\left(1 / \Psi\left(\lambda_{s}^{1, l}\left(\rho^{*}\right)\right)\right)}{1 / \lambda_{s}^{1, l}\left(\rho^{*}\right)-1 / \Psi\left(\lambda_{s}^{1, l}\left(\rho^{*}\right)\right)}=\frac{\tilde{p}\left(1 / \lambda_{s}^{1, l}\left(\rho^{*}\right)\right)-\tilde{p}\left(1 / \rho^{*}\right)}{1 / \lambda_{s}^{1, l}\left(\rho^{*}\right)-1 / \rho^{*}}=\tilde{p}_{\tau}\left(\tau^{*}\right),
$$




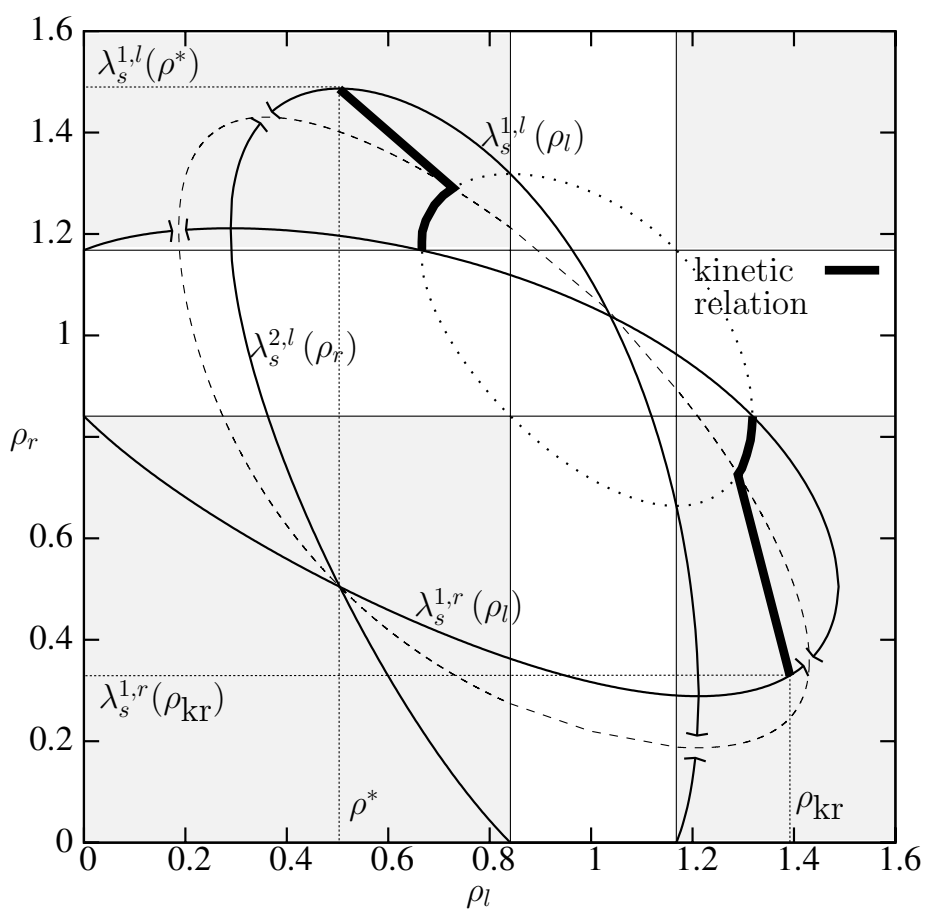

Figure 8. The graph of the kinetic relation (bold solid line) is displayed as given in Definition 2.12 is displayed. Furthermore the entropy dissipation curve $\Gamma_{\Delta}$ (dashed line), and the characteristic curves (solid lines) are shown which impose some restrictions on the choice of the kinetic relation as discussed in Remark 2.13.

due to the definition of $\lambda_{s}^{1, l}$ and

$$
h_{1}\left(1 / \rho_{\text {liquid }}^{M}\right)=\frac{\tilde{p}\left(1 / \rho_{\text {liquid }}^{M}\right)-\tilde{p}\left(1 / \Psi\left(\rho_{\text {liquid }}^{M}\right)\right)}{1 / \rho_{\text {liquid }}^{M}-1 / \Psi\left(\rho_{\text {liquid }}^{M}\right)}=\frac{\tilde{p}\left(1 / \rho_{\text {liquid }}^{M}\right)-\tilde{p}\left(1 / \rho_{\text {vapor }}^{M}\right)}{1 / \rho_{\text {liquid }}^{M}-1 / \rho_{\text {vapor }}^{M}}=0 .
$$

A short calculation using (4) (cf. Assumption 1.1) and the monotonicity of $\Psi$ (48) shows

$$
\frac{\mathrm{d} h_{1}}{\mathrm{~d} \tau}(\tau)>0
$$

On the other hand for all $\rho_{l} \in\left(0, \rho_{\text {min }}^{\text {ellipt }}\right)$ we have

$$
\begin{gathered}
h_{2, l}\left(1 / \lambda_{s}^{1, l}\left(\rho^{*}\right)\right)=\frac{\tilde{p}\left(1 / \rho_{l}\right)-\tilde{p}\left(1 / \Psi\left(\lambda_{s}^{1, l}\left(\rho^{*}\right)\right)\right)}{1 / \rho_{l}-1 / \Psi\left(\lambda_{s}^{1, l}\left(\rho^{*}\right)\right)}=\frac{\tilde{p}\left(1 / \rho_{l}\right)-\tilde{p}\left(1 / \rho^{*}\right)}{1 / \rho_{l}-1 / \rho^{*}}>\tilde{p}_{\tau}\left(\tau^{*}\right), \\
h_{2, l}\left(1 / \rho_{\text {liquid }}^{M}\right)=\frac{\tilde{p}\left(1 / \rho_{l}\right)-\tilde{p}\left(1 / \Psi\left(\rho_{\text {liquid }}^{M}\right)\right)}{1 / \rho_{l}-1 / \Psi\left(\rho_{\text {liquid }}^{M}\right)}=\frac{\tilde{p}\left(1 / \rho_{l}\right)-\tilde{p}\left(1 / \rho_{\text {vapor }}^{M}\right)}{1 / \rho_{l}-1 / \rho_{\text {vapor }}^{M}}<0,
\end{gathered}
$$

and with the same arguments as above

$$
\frac{\mathrm{d} h_{2, l}}{\mathrm{~d} \tau}(\tau)<0
$$


Therefore, there is exactly one $\hat{\tau}_{l} \in\left(1 / \lambda_{s}^{1, l}\left(\rho^{*}\right), 1 / \rho_{\text {liquid }}^{M}\right)$ such that $h_{1}\left(\hat{\tau}_{l}\right)=h_{2, l}\left(\hat{\tau}_{l}\right)$. Now the statement follows from

$$
g\left(\rho_{l}\right):=\hat{\tau}_{l}^{-1} \in\left(\rho_{\text {liquid }}^{M}, \lambda_{s}^{1, l}\left(\rho^{*}\right)\right)
$$

Now we proceed to the main theorem recalling that we denote by a classical $k$-wave a $k$-shock/rarefaction/attached wave.

Theorem 3.2 (Generalized 1-wave curve). Let $v_{l} \in \mathbb{R}$ and $\rho_{l} \in \mathcal{A}$.

Case 1. $\rho_{l} \in\left(0, \rho^{*}\right]$ :

- For $\rho_{r} \in\left(0, \rho_{\mathrm{min}}^{\mathrm{ellipt}}\right)$ there exists a unique $v_{r} \in \mathbb{R}$, such that $\left(\rho_{l}, v_{l}\right)$ can be connected to $\left(\rho_{r}, v_{r}\right)$ by a classical 1-wave.

- For $\rho_{r} \in\left(\rho_{\max }^{\text {ellipt }}, g\left(\rho_{l}\right)\right]$ :

- If $\Psi\left(\rho_{r}\right) \in\left(\lambda_{s}^{1, r}\left(\rho_{l}\right), \rho_{\mathrm{min}}^{\mathrm{ellipt}}\right)$ there exists a unique $v_{r} \in \mathbb{R}$, such that $\left(\rho_{l}, v_{l}\right)$ can be connected to $\left(\rho_{r}, v_{r}\right)$ by an 1 -attached wave $\left(\rho_{l}, v_{l}\right) \stackrel{\text { att }}{\rightarrow}\left(\Psi\left(\rho_{r}\right), v\right)$ for some $v \in \mathbb{R}$ followed by a phase transition $\left(\Psi\left(\rho_{r}\right), v\right) \stackrel{p t}{\rightarrow}\left(\rho_{r}, v_{r}\right)$.

- If $\Psi\left(\rho_{r}\right) \in\left(\rho^{*}, \lambda_{s}^{1, r}\left(\rho_{l}\right)\right)$ : there exists a unique $v_{r} \in \mathbb{R}$, such that $\left(\rho_{l}, v_{l}\right)$ can be connected to $\left(\rho_{r}, v_{r}\right)$ by a Laxian 1-shock wave $\left(\rho_{l}, v_{l}\right) \stackrel{s}{\rightarrow}\left(\Psi\left(\rho_{r}\right), v\right)$ for some $v \in \mathbb{R}$ followed by a phase transition $\left(\Psi\left(\rho_{r}\right), v\right) \stackrel{p t}{\rightarrow}\left(\rho_{r}, v_{r}\right)$.

- For $\rho_{r} \in\left(g\left(\rho_{l}\right), 1 / b\right)$ there exists a unique $v_{r} \in \mathbb{R}$, such that $\left(\rho_{l}, v_{l}\right)$ can be connected to $\left(\rho_{r}, v_{r}\right)$ by a Laxian 1-shock wave.

Case 2. $\rho_{l} \in\left(\rho^{*}, \rho_{\min }^{\text {ellipt }}\right)$ :

- For $\rho_{r} \in\left(0, \rho_{\mathrm{min}}^{\text {ellipt }}\right)$ there exists a unique $v_{r} \in \mathbb{R}$, such that $\left(\rho_{l}, v_{l}\right)$ can be connected to $\left(\rho_{r}, v_{r}\right)$ by a classical 1-wave.

- For $\rho_{r} \in\left(\rho_{\max }^{\text {ellipt }}, g\left(\rho_{l}\right)\right]$ :

- If $\Psi\left(\rho_{r}\right) \in\left(\rho_{l}, \rho_{\mathrm{min}}^{\text {ellipt }}\right)$ there exists a unique $v_{r} \in \mathbb{R}$, such that $\left(\rho_{l}, v_{l}\right)$ can be connected to $\left(\rho_{r}, v_{r}\right)$ by an 1-rarefaction wave $\left(\rho_{l}, v_{l}\right) \stackrel{\text { rare }}{\rightarrow}\left(\Psi\left(\rho_{r}\right), v\right)$ for some $v \in \mathbb{R}$ followed by a phase transition $\left(\Psi\left(\rho_{r}\right), v\right) \stackrel{p t}{\longrightarrow}\left(\rho_{r}, v_{r}\right)$.

- If $\Psi\left(\rho_{r}\right) \in\left(\rho^{*}, \rho_{l}\right)$ there exists a unique $v_{r} \in \mathbb{R}$, such that $\left(\rho_{l}, v_{l}\right)$ can be connected to $\left(\rho_{r}, v_{r}\right)$ by a Laxian 1-shock wave $\left(\rho_{l}, v_{l}\right) \stackrel{s}{\rightarrow}\left(\Psi\left(\rho_{r}\right), v\right)$ for some $v \in \mathbb{R}$ followed by a phase transition $\left(\Psi\left(\rho_{r}\right), v\right) \stackrel{p t}{\longrightarrow}\left(\rho_{r}, v_{r}\right)$.

- For $\rho_{r} \in\left(g\left(\rho_{l}\right), 1 / b\right)$ there exists a unique $v_{r} \in \mathbb{R}$, such that $\left(\rho_{l}, v_{l}\right)$ can be connected to $\left(\rho_{r}, v_{r}\right)$ by a Laxian 1-shock wave.

Case 3. $\rho_{l} \in\left(\rho_{\max }^{\text {ellipt }}, 1 / b\right)$ :

- For $\rho_{r} \in\left(0, \lambda_{s}^{1, r}\left(\rho_{k r}\right)\right)$ :

- If $\rho_{l} \in\left(\rho_{k r}, 1 / b\right)$ there exists a unique $v_{r} \in \mathbb{R}$, such that $\left(\rho_{l}, v_{l}\right)$ can be connected to $\left(\rho_{r}, v_{r}\right)$ by an 1-rarefaction wave $\left(\rho_{l}, v_{l}\right) \stackrel{\text { rare }}{\longrightarrow}\left(\rho_{k r}, v_{k r}\right)$ for some $v_{k r} \in \mathbb{R}$ followed by a phase transition $\left(\rho_{k r}, v_{k r}\right) \stackrel{p t}{\longrightarrow}\left(\lambda_{s}^{1, r}\left(\rho_{k r}\right), v\right)$ for some $v \in \mathbb{R}$ followed by an attached 1-rarefaction wave $\left(\lambda_{s}^{1, r}\left(\rho_{k r}\right), v\right) \stackrel{\text { rare }}{\longrightarrow}\left(\rho_{r}, v_{r}\right)$.

- If $\rho_{l} \in\left(\rho_{\max }^{\mathrm{ellipt}}, \rho_{k r}\right)$ there exists a unique $v_{r} \in \mathbb{R}$, such that $\left(\rho_{l}, v_{l}\right)$ can be connected to $\left(\rho_{r}, v_{r}\right)$ by a Laxian 1-shock wave $\left(\rho_{l}, v_{l}\right) \stackrel{s}{\rightarrow}\left(\rho_{k r}, v_{k r}\right)$ followed by a phase transition $\left(\rho_{k r}, v_{k r}\right) \stackrel{p t}{\rightarrow}\left(\lambda_{s}^{1, r}\left(\rho_{k r}\right), v\right)$ for some $v \in \mathbb{R}$ followed by an attached 1 -rarefaction wave $\left(\lambda_{s}^{1, r}\left(\rho_{k r}\right), v\right) \stackrel{\text { rare }}{\longrightarrow}\left(\rho_{r}, v_{r}\right)$. 


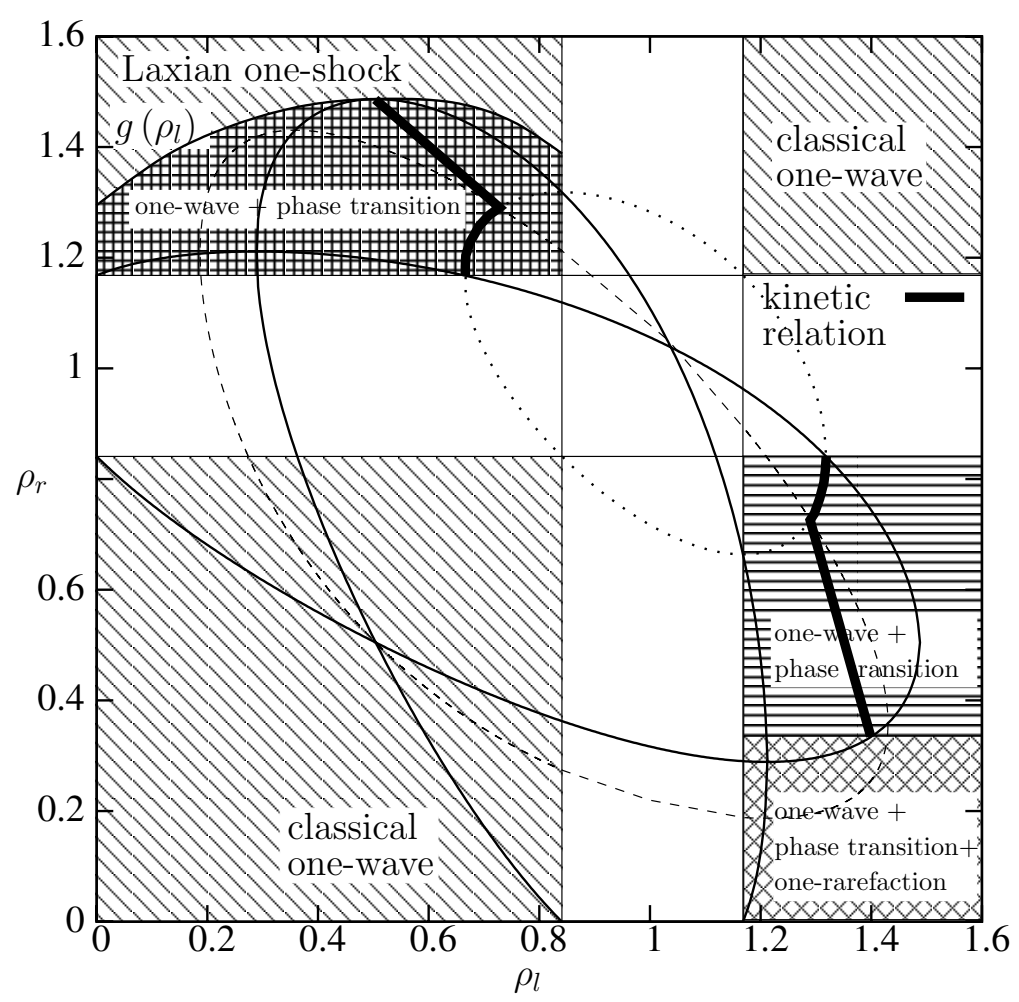

Figure 9. Structure of the generalized 1-wave curve ( $c f$. Def. 3.2). Combinations of states $\rho_{l}$ and $\rho_{r}$ can be connected by a combination of rarefaction-waves, Laxian shock-waves and undercompressive shock-waves satisfying the kinetic relation.

- For $\rho_{r} \in\left(\lambda_{s}^{1, r}\left(\rho_{k r}\right), \rho_{\min }^{\text {ellipt }}\right)$ :

- If $\rho_{l} \in\left(\Psi\left(\rho_{r}\right), 1 / b\right)$ there exists a unique $v_{r} \in \mathbb{R}$, such that $\left(\rho_{l}, v_{l}\right)$ can be connected to $\left(\rho_{r}, v_{r}\right)$ by an 1-rarefaction wave $\left(\rho_{l}, v_{l}\right) \stackrel{\text { rare }}{\rightarrow}\left(\Psi\left(\rho_{r}\right), v\right)$ for some $v \in \mathbb{R}$ followed by a phase transition $\left(\Psi\left(\rho_{r}\right), v\right) \stackrel{p t}{\rightarrow}\left(\rho_{r}, v_{r}\right)$.

- If $\rho_{l} \in\left(\rho_{\max }^{\text {ellipt }}, \Psi\left(\rho_{r}\right)\right)$ there exists a unique $v_{r} \in \mathbb{R}$, such that $\left(\rho_{l}, v_{l}\right)$ can be connected to $\left(\rho_{r}, v_{r}\right)$ by a Laxian 1-shock wave $\left(\rho_{l}, v_{l}\right) \stackrel{s}{\rightarrow}\left(\Psi\left(\rho_{r}\right), v\right)$ for some $v \in \mathbb{R}$ followed by a phase transition $\left(\Psi\left(\rho_{r}\right), v\right) \stackrel{p t}{\rightarrow}\left(\rho_{r}, v_{r}\right)$.

- If $\rho_{r} \in\left(\rho_{\max }^{\text {ellipt }}, 1 / b\right)$ there exists a unique $v_{r} \in \mathbb{R}$, such that $\left(\rho_{l}, v_{l}\right)$ can be connected to $\left(\rho_{r}, v_{r}\right)$ by a classical 1-wave.

All shock waves are entropy-dissipative.

Proof. The existence and uniqueness and thermodynamical admissibility of the constructions above follows from Lemmas 2.6, 2.9, 2.10, the definition of the kinetic relation (Def. 2.12) and Remark 2.13.

For the well-posedness of the constructions above we have to check that in each case the elementary waves do not interact:

Case 1. $\rho_{l} \in\left(0, \rho^{*}\right]:$

For $\rho_{r} \in\left(0, \rho_{\text {min }}^{\text {ellipt }}\right)$ the solution consists of a single classical elementary wave ( $c f$. Lems. 2.6, 2.9 and 2.10). 
For $\rho_{r} \in\left(\rho_{\max }^{\text {ellipt }}, g\left(\rho_{l}\right)\right)$ and $\Psi\left(\rho_{r}\right) \in\left(\lambda_{s}^{1, r}\left(\rho_{l}\right), \rho_{\min }^{\text {ellipt }}\right):$ The rightmost speed of the 1-attached wave $\left(\rho_{l}, v_{l}\right) \stackrel{\text { att }}{\rightarrow}$ $\left(\Psi\left(\rho_{r}\right), v\right)$ for some $v \in \mathbb{R}\left(c f\right.$. Lem. 2.10) is smaller than the speed of the succeeding phase transition $\left(\Psi\left(\rho_{r}\right), v\right) \stackrel{p t}{\rightarrow}$ $\left(\rho_{r}, v_{r}\right)$ because of $(30)$. So this construction is always $\Psi$-admissible.

If $\rho_{r} \in\left(\rho_{\max }^{\text {ellipt }}, g\left(\rho_{l}\right)\right)$ and $\Psi\left(\rho_{r}\right) \in\left(\rho^{*}, \lambda_{s}^{1, r}\left(\rho_{l}\right)\right):$ The speed $s$ of the preceding 1-shock wave $\left(\rho_{l}, v_{l}\right) \stackrel{s}{\rightarrow}$ $\left(\Psi\left(\rho_{r}\right), v\right)$ for some $v \in \mathbb{R}$ has to be smaller than the speed $s_{\mathrm{ph}}$ of the following phase transition $\left(\Psi\left(\rho_{r}\right), v\right) \stackrel{p t}{\rightarrow}$ $\left(\rho_{r}, v_{r}\right)$ :

$$
\underbrace{v-\left(\frac{\rho_{l}}{\Psi\left(\rho_{r}\right)} \frac{p\left(\Psi\left(\rho_{r}\right)\right)-p\left(\rho_{l}\right)}{\Psi\left(\rho_{r}\right)-\rho_{l}}\right)^{\frac{1}{2}}}_{S}<\underbrace{v-\left(\frac{\rho_{r}}{\Psi\left(\rho_{r}\right)} \frac{p\left(\rho_{r}\right)-p\left(\Psi\left(\rho_{r}\right)\right)}{\rho_{r}-\Psi\left(\rho_{r}\right)}\right)^{\frac{1}{2}}}_{s_{\mathrm{ph}}} .
$$

Using the Lagrangian pressure $\tilde{p}$ this is equivalent to

$$
\frac{\tilde{p}\left(\tau_{l}\right)-\tilde{p}(\hat{\tau})}{\tau_{l}-\hat{\tau}}>\frac{\tilde{p}\left(\tau_{r}\right)-\tilde{p}(\hat{\tau})}{\tau_{r}-\hat{\tau}}
$$

where $\hat{\tau}=1 / \Psi\left(\rho_{r}\right)$. Because of Lemma 3.1 this is true for

$$
\rho_{r}<g\left(\rho_{l}\right)
$$

For $\rho_{r}=g\left(\rho_{l}\right): s=s_{\mathrm{ph}}$ and $\left(\rho_{r}, v_{r}\right) \stackrel{s}{\rightarrow}\left(\rho_{l}, v_{l}\right)$ can be connected by a single Laxian shock with speed $s$.

Finally, for all $\rho_{r}>g\left(\rho_{l}\right)$ the left and right hand state are connected by a single Laxian shock wave $\left(\rho_{r}, v_{r}\right) \stackrel{s}{\rightarrow}$ $\left(\rho_{l}, v_{l}\right)$.

Case $\mathbf{2}$ is checked similarly.

Case 3. $\rho_{l} \in\left(\rho_{\max }^{\text {ellipt }}, 1 / b\right)$ :

For simplicity suppose $\rho_{r} \in\left(0, \lambda_{s}^{1, r}\left(\rho_{k r}\right)\right)$. The left-hand state $\left(\rho_{l}, v_{l}\right)$ is connected to $\left(\rho_{k r}, v_{k r}\right)$ for some $v_{k r}$ by a classical 1-wave (either a shock or a rarefaction wave). Then, $\left(\rho_{k r}, v_{k r}\right) \stackrel{p t}{\rightarrow}\left(\lambda_{s}^{1, r}\left(\rho_{k r}\right), v\right)$ are joined by a $\Psi$-admissible phase transition. Finally, $\left(\lambda_{s}^{1, r}\left(\rho_{k r}\right), v\right) \stackrel{\text { rare }}{\rightarrow}\left(\rho_{r}, v_{r}\right)$ is connected to each other by a rarefaction wave.

Again we have to check the speeds of the waves: Suppose the leftmost wave is a rarefaction wave then the fastest speed of propagation is $\lambda_{1}\left(\rho_{k r}, v_{k r}\right)$ which is less than the speed of the undercompressive shock wave due to (30).

If the leftmost wave is a classical shock wave then this property can be calculated with the help of (19).

The rightmost wave always is a rarefaction wave. The smallest speed of propagation is $\lambda_{1}\left(\lambda_{s}^{1, r}\left(\rho_{k r}\right), v\right)$ which equals the speed of the preceding undercompressive wave due to the definition of $\lambda_{s}^{1, r}$.

With Theorem 3.2 there is defined the generalized 1-wave curve, i.e., the set consisting of all the right handstates $\left(\rho_{r}, v_{r}\right) \in \mathcal{A} \times \mathbb{R}$ that can be connected to a left hand state $\left(\rho_{l}, v_{l}\right) \in \mathcal{A} \times \mathbb{R}$ by a combination of 1-rarefaction waves, Laxian 1-shock waves, 1-attached waves, and undercompressive shock waves satisfying the kinetic relation (47) ( $c f$. Fig. 9). In Figure 10a a the generalized 1-wave curve is depicted for a specific choice of $\left(\rho_{l}, v_{l}\right)$.

From the construction of the generalized 1-wave curve in Theorem 3.2 it is clear that the righthand velocity state can be computed uniquely and continuously from the given state $\left(\rho_{l}, v_{l}\right)$ and the righthand density state $\rho_{r} \in\left(0, \rho_{\min }^{\text {ellipt }}\right) \cup\left(\rho_{\max }^{\text {ellipt }}, 1 / b\right)$. Let us therefore define the mapping

$$
W_{1}^{\rho_{l}, v_{l}}:\left\{\begin{aligned}
\left(0, \rho_{\min }^{\text {ellipt }}\right) \cup\left(\rho_{\max }^{\text {ellipt }}, 1 / b\right) & \longrightarrow \mathbb{R} \\
\rho & \longmapsto v_{r}(\rho),
\end{aligned}\right.
$$

where $v_{r}=v_{r}(\rho)$ is this righthand velocity state. 


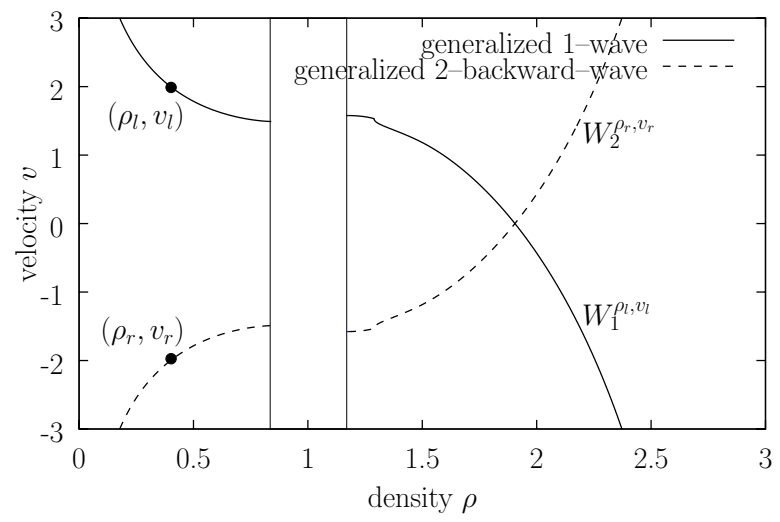

(a) Generalized 1-wave curve (cf. Thm. 3.2) and generalized 2-backward-wave curve (Thm. 3.4).

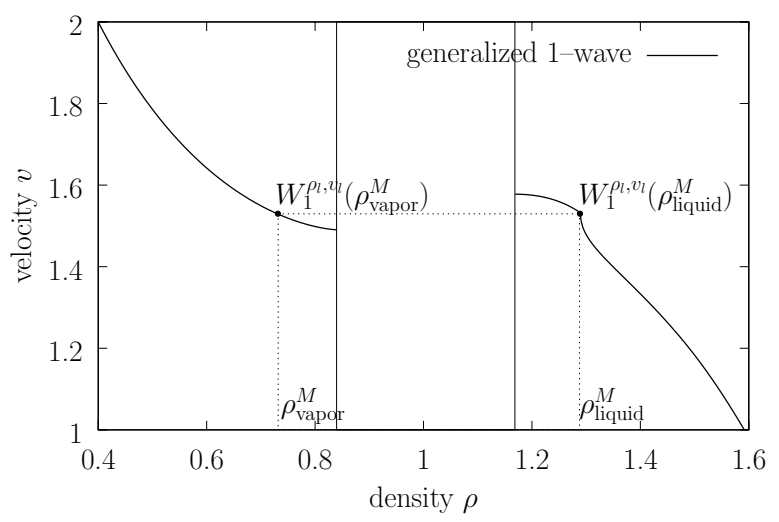

(b) Zoom into the generalized one-wave curve from Figure 10a.

FigurE 10. Example of generalized wave curves for the states $\rho_{l}=\rho_{r}=0.4, v_{l}=-v_{r}=2$.

Lemma 3.3. The function $W_{1}^{\rho_{l}, v_{l}}$ from (49) is monotone decreasing. We have

$$
\lim _{\rho \rightarrow 0} W_{1}^{\rho_{l}, v_{l}}(\rho)=\infty \quad \text { and } \quad \lim _{\rho \rightarrow 1 / b} W_{1}^{\rho_{l}, v_{l}}(\rho)=-\infty
$$

Further, it holds

$$
W_{1}^{\rho_{l}, v_{l}}\left(\rho_{\min }^{\text {ellipt }}\right)<W_{1}^{\rho_{l}, v_{l}}\left(\rho_{\max }^{\text {ellipt }}\right)
$$

Proof. Let $\left(\rho_{l}, v_{l}\right) \in\left(0, \rho_{\min }^{\text {ellipt }}\right) \cup\left(\rho_{\max }^{\text {ellipt }}, 1 / b\right)$, then if $\rho_{r}$ is small enough the rightmost wave of the generalized 1 -wave is a 1 -rarefaction wave from some $(\hat{\rho}, \hat{v}) \in\left(0, \rho_{\min }^{\text {ellipt }}\right) \times \mathbb{R}$ to $\left(\rho_{r}, v_{r}\right)$ and

$$
\lim _{\rho_{r} \rightarrow 0} v_{r}\left(\rho_{r}\right)=\lim _{\rho_{r} \rightarrow 0}\left(\hat{v}-\int_{\hat{\rho}}^{\rho_{r}} \frac{\sqrt{p^{\prime}(\rho)}}{\rho} \mathrm{d} \rho\right)>\lim _{\rho_{r} \rightarrow 0}\left(\hat{v}-\sqrt{p^{\prime}(\hat{\rho})} \int_{\hat{\rho}}^{\rho_{r}} \frac{1}{\rho} \mathrm{d} \rho\right) \longrightarrow \infty
$$

On the other hand if $\rho_{r} \rightarrow 1 / b$ the left and right states will be connected by a Laxian 1-shock wave if $\rho_{r}$ is big enough and

$$
\lim _{\rho_{r} \rightarrow 1 / b} v_{r}\left(\rho_{r}\right)=\lim _{\rho_{r} \rightarrow 1 / b}\left(v_{l}-\left(\frac{\rho_{r}-\rho_{l}}{\rho_{l} \rho_{r}}\left(p\left(\rho_{r}\right)-p\left(\rho_{l}\right)\right)\right)^{\frac{1}{2}}\right) \longrightarrow-\infty
$$

The monotonicity of the generalized 1-wave curve is a consequence of the monotonicity properties of the elementary waves ( $c f$. Rems. 2.2, 2.8 and 2.11) as the generalized 1-wave is composed of elementary waves.

Now it remains to show $W_{1}^{\rho_{l}, v_{l}}\left(\rho_{\min }^{\text {ellipt }}\right)<W_{1}^{\rho_{l}, v_{l}}\left(\rho_{\max }^{\text {ellipt }}\right)$ : Let $\left(\rho_{l}, v_{l}\right) \in \mathcal{A} \times \mathbb{R}$ be given and suppose $\rho_{l} \in$ $\left(0, \rho_{\min }^{\text {ellipt }}\right)$. The case $\rho_{l} \in\left(\rho_{\max }^{\text {ellipt }}, 1 / b\right)$ is proved analogously.

It suffices to prove $W_{1}^{\rho_{l}, v_{l}}\left(\rho_{\text {vapor }}^{M}\right)=W_{1}^{\rho_{l}, v_{l}}\left(\rho_{\text {liquid }}^{M}\right)$ and to use the monotonicity of $W_{1}^{\rho_{l}, v_{l}}$ in each phase (see Fig. 10b). $\rho_{\text {liquid }}^{M}$ is reached by a classical wave $\rho_{l} \longrightarrow \rho_{\text {vapor }}^{M}$ and an undercompressive shock wave $\rho_{\text {vapor }}^{M} \longrightarrow$ $\rho_{\text {liquid }}^{M}$ connecting the Maxwell states. It is easy to check from the Rankine-Hugoniot conditions (14) that since $p\left(\rho_{\text {vapor }}^{M}\right)=p\left(\rho_{\text {liquid }}^{M}\right)$ the velocities on both sides of the shock wave are the same and therefore, $W_{1}^{\rho_{l}, v_{l}}\left(\rho_{\text {vapor }}^{M}\right)=$ $W_{1}^{\rho_{l}, v_{l}}\left(\rho_{\text {liquid }}^{M}\right)$. 


\subsection{The generalized 2-backward-wave curve}

Similar results as in Theorem 3.2 and Lemma 3.3 can be shown for the two-wave curve. Since we want to solve finally the Riemann problem we are interested in the so-called two-backward-wave curve, i.e., the set consisting of all the left hand states $\left(\rho_{l}, v_{l}\right) \in \mathcal{A} \times \mathbb{R}$ that can be connected to a given right hand state $\left(\rho_{r}, v_{r}\right) \in \mathcal{A} \times \mathbb{R}$ by a combination of elementary waves.

Theorem 3.4 (Generalized 2-backward-wave curve). Let a righthand state $\left(\rho_{r}, v_{r}\right) \in \mathcal{A} \times \mathbb{R}$ be given. The set of all lefthand states $\left(\rho_{l}, v_{l}\right) \mathcal{A} \times \mathbb{R}$ that can be connected by a combination of 2-rarefaction waves, Laxian 2-shock waves, 2-attached waves, and $\Psi$-admissible phase transitions is given by $\left\{\left(\rho, W_{2}^{\rho_{r}, v_{r}}(\rho)\right) \mid \rho \in \mathcal{A}\right\}$ where $W_{2}^{\rho_{r}, v_{r}}$ is defined through

$$
W_{2}^{\rho_{r}, v_{r}}(\rho)=-W_{1}^{\rho_{r},-v_{r}}(\rho) \quad(\rho \in \mathcal{A}) .
$$

The shock waves are entropy-dissipative.

Proof. As the generalized wave curves are a combination of shock - and rarefaction - waves, it is enough to show the statement for those waves.

In the case of a rarefaction wave: Let $\left(\rho_{l}, v_{l}\right) \mathcal{A} \times \mathbb{R}$ and $\left(\rho_{r}, v_{r}\right) \mathcal{A} \times \mathbb{R}$ two states that can be connected by a 2-rarefaction wave ( $c f$. Lem. 2.9). Then, we can calculate

$$
W_{2}^{\rho_{r}, v_{r}}\left(\rho_{l}\right)=v_{r}-\int_{\rho_{l}}^{\rho_{r}} \frac{\sqrt{p^{\prime}(\rho)}}{\rho} \mathrm{d} \rho
$$

and

$$
-W_{1}^{\rho_{r},-v_{r}}\left(\rho_{l}\right)=-\left(-v_{r}-\int_{\rho_{r}}^{\rho_{l}} \frac{\sqrt{p^{\prime}(\rho)}}{\rho} \mathrm{d} \rho\right)=v_{r}-\int_{\rho_{l}}^{\rho_{r}} \frac{\sqrt{p^{\prime}(\rho)}}{\rho} \mathrm{d} \rho=W_{2}^{\rho_{r}, v_{r}}\left(\rho_{l}\right) .
$$

The same calculation can be done for shock waves using Lemma 2.6 and (18).

To observe that shock waves in the 2-backward curve are entropy-dissipative consider (24). The sign of $j$ changes if we switch from a 1-shock wave to a 2-shock wave. Since we have also interchanged the role of $\rho_{l}$ and $\rho_{r}$ in $(51)$ the overall sign in $(24)$ does not change. Note that $\Delta\left(\rho_{r}, \rho_{l}\right)=\Delta\left(\rho_{l}, \rho_{r}\right)$ holds.

From Lemma 3.3 we directly get

Lemma 3.5. The function $W_{2}^{\rho_{r}, v_{r}}$ from (51) is a continuous, monotone increasing function. It holds

$$
\lim _{\rho \rightarrow 0} W_{1}^{\rho_{l}, v_{l}}(\rho)=-\infty \quad \text { and } \quad \lim _{\rho \rightarrow 1 / b} W_{1}^{\rho_{l}, v_{l}}(\rho)=\infty,
$$

and further

$$
W_{2}^{\rho_{r}, v_{r}}\left(\rho_{\min }^{\text {ellipt }}\right)>W_{2}^{\rho_{r}, v_{r}}\left(\rho_{\max }^{\text {ellipt }}\right)
$$

\subsection{Thermodynamically admissible solution of the Riemann problem}

The Riemann problem (1), (3) can now be solved if one analyzes whether there are points $(\hat{\rho}, \hat{v}) \in \mathcal{A} \times \mathbb{R}$ such that we have

$$
W_{1}^{\rho_{L}, v_{L}}(\hat{\rho})=W_{2}^{\rho_{R}, v_{R}}(\hat{\rho})=\hat{v}
$$

From Theorems 3.2 and 3.4 we can then explicitly construct the weak solutions. It will turn out that the intersection points $(\hat{\rho}, \hat{v})$ are not unique in general. The subsequent theorem summarizes our result and in particular gives physically reasonable criteria for a unique solution.

Theorem 3.6 (Existence and uniqueness for Riemann problem). Consider the Riemann problem (1), (3) where the pressure is given by (10). Let $\Psi$ be given by Definition (2.12).

(i) The Riemann problem admits a weak solution in the class of self-similar functions consisting of Laxian shock waves, $\Psi$-admissible phase transitions, attached and rarefaction waves. 
(ii) It is unique in this class if it satisfies the following properties:

(1) phase transitions connecting states with the same pressure connect Maxwell-states $\rho_{\text {vapor }}^{M}$ and $\rho_{\text {liquid }}^{M}$;

(2) a one-phase solution is used whenever it exists.

The shock waves within these weak solutions are entropy-dissipative.

Proof. Due to (50), (52) and the monotonicity of $W_{1}^{\rho_{L}, v_{L}}$ and $W_{2}^{\rho_{R}, v_{R}}$ described in Lemmas 3.3 and 3.5 there exist at least one and at most two intersection points $\left(\hat{\rho}_{1 / 2}, \hat{v}_{1 / 2}\right) \in \mathcal{A} \times \mathbb{R}$ of $W_{1}^{\rho_{L}, v_{L}}$ and $W_{2}^{\rho_{R}, v_{R}}$, i.e., solutions of (53). Thus we have proven (i).

If there is only one intersection point, say $\left(\hat{\rho}_{1}, \hat{v}_{1}\right)$ there is a unique solution of the Riemann problem consisting of a combination of 1-waves from $\left(\rho_{L}, v_{L}\right)$ to $\left(\hat{\rho}_{1}, \hat{v}_{1}\right)\left(c f\right.$. Thm. 3.2) and a combination of 2-waves from $\left(\hat{\rho}_{1}, \hat{v}_{1}\right)$ to $\left(\rho_{R}, v_{R}\right)(c f$. Thm. 3.4).

If there are two intersection points $\left(\hat{\rho}_{1}, \hat{v}_{1}\right)$ and $\left(\hat{\rho}_{2}, \hat{v}_{2}\right)$ - thus two solutions - they are located in different phases. We have to eliminate exactly one of them.

Case 1. $\rho_{L}$ and $\rho_{R}$ are also located in different phases. Suppose for definiteness that $\rho_{L} \in\left(0, \rho_{\text {min }}^{\text {ellipt }}\right)$ and $\rho_{R} \in\left(\rho_{\max }^{\text {ellipt }}, 1 / b\right)$ holds.

Assume that for the first intersection point $\hat{\rho}_{1}<\rho_{\text {vapor }}^{M}$ holds. Then, due to the kinetic relation (47) for the according phase transition it holds $p\left(\hat{\rho}_{1}\right) \neq p\left(\Psi\left(\hat{\rho}_{1}\right)\right)$.

Moreover, due to the monotonicity of the wave curves the following equation is valid:

$$
W_{1}^{\rho_{L}, v_{L}}\left(\rho_{\text {liquid }}^{M}\right)=W_{1}^{\rho_{L}, v_{L}}\left(\rho_{\text {vapor }}^{M}\right)<W_{1}^{\rho_{L}, v_{L}}\left(\hat{\rho}_{1}\right)=W_{2}^{\rho_{R}, v_{R}}\left(\hat{\rho}_{1}\right)<W_{2}^{\rho_{R}, v_{R}}\left(\rho_{\text {vapor }}^{M}\right)=W_{2}^{\rho_{R}, v_{R}}\left(\rho_{\text {liquid }}^{M}\right) .
$$

Due to the monotonicity of $W_{1}^{\rho_{L}, v_{L}}$ and $W_{2}^{\rho_{R}, v_{R}}$ it holds for the second intersection point

$$
\hat{\rho}_{2}<\rho_{\text {liquid }}^{M}
$$

and due to the kinetic relation (47)

$$
p\left(\hat{\rho}_{2}\right)=p\left(\Psi\left(\hat{\rho}_{2}\right)\right) .
$$

Thus the solution associated with the second intersection point is excluded by condition (1).

For $\hat{\rho}_{1}>\rho_{\text {vapor }}^{M}$ we have $p\left(\hat{\rho}_{1}\right)=p\left(\rho_{\text {vapor }}^{M}\right)$. The argument as above shows then $p\left(\hat{\rho}_{2}\right) \neq p\left(\rho_{\text {vapor }}^{M}\right)$ which is chosen by condition (1).

Case 2. $\rho_{L}$ and $\rho_{R}$ are located within a single phase.

If there are two intersection points one of them is located within the same phase as $\rho_{L}$ and $\rho_{R}$ and we get a unique solution within one phase by condition (2).

The shock waves in the constructed solution do satisfy (22) since it is constructed by a combination of the thermodynamically admissible waves described in Theorems 3.2 and 3.4 .

One can check that there are Riemann problems with states in the same phase such that the solutions constructed in Theorem 3.6 contain density values in different phases: nucleation occurs. The condition (2) in Theorem 3.6 can be seen as a nucleation criterium for a new phase. We suppose that the fluid does not tend to nucleate a new phase if possible. Also other less restrictive criteria can be used to enforce uniqueness. It is out of the scope of this paper to prove rigorously the $L^{1}$-continuous dependence of our Riemann-solver with respect to initial data. In particular this has to be shown for the critical case of nucleation. Results in this direction can be found in [9] for similar models.

Condition (1) can be motivated e.g. by work from statistical mechanics. Starting from an equilibrium particle set-up in the sense of statistical mechanics a formal thermodynamical limit procedure shows that the limit density distribution leads to Maxwell states [19]. Therefore we exclude all stationary phase transitions, i.e. connections with the same pressure on both sides, which do not connect Maxwell states. 


\section{A Riemann solver based ghost fluid method}

In this chapter we present a numerical scheme for the mixed hyperbolic-elliptic Cauchy-problem (1), (2) with non-monotone equation of state. In principle one could apply a "classical" finite-volume (FV) scheme to get a numerical solution $(c f .[15])$. However, these schemes will typically fail for a hyperbolic-elliptic problem for two reasons:

- First, due to artificial dissipation shock waves in the solution will be smeared out. In the case of a phase transition a smeared out wave will eventually contain values of $\rho$ that are situated within the elliptic region. Most of the above schemes will abort then since e.g. the term $\sqrt{p^{\prime}(\rho)}$ contained in the calculation of the eigenvalues (11) is not defined as a real number.

- Second, for schemes that will not break down (e.g. Lax-Friedrichs) and might even converge to an entropy solution it cannot be guarented that the limit will satisfy a given kinetic relation. Since the entropy solution depends sensitively on the kinetic relation not used in the schemes spurious solutions show up.

These issues can be avoided if one tracks carefully the position and motion of all phase boundaries and represent them as sharp fronts. The movement of sharp discontinuities can be controlled by a level set method (see e.g. $[29,32,34,37])$. Then at the interface we use an exact Riemann solver of the problem (see Sect. 3) which includes the kinetic relation and keeps the phase boundary discontinuous. The idea of this scheme is similar to the class of Ghost Fluid schemes [13,14,26]. They address multi-material flows without mass transfer between the different materials and only approximate Riemann solvers are used. In the sequel we assume that the Cauchy problem (1), (2) with

$$
\mathbf{w}_{0}:=\left(\rho_{0}, m_{0}\right)^{T} \in L_{l o c}^{1}(\mathbb{R}, \mathcal{A} \times \mathbb{R})
$$

admits a unique weak solution $\mathbf{w}:=\left(\rho, m^{T}\right) \in L_{l o c}^{1}(\mathbb{R} \times[0, \infty), \mathcal{A} \times \mathbb{R})$ such that all shock waves are entropydissipative and such that all phase transitions are $\Psi$-admissible with $\Psi$ given in Definition 2.12.

To motivate the new algorithm we introduce an initial value problem for a level set equation which is used in the algorithm. Let $\varphi_{0}: \mathbb{R} \rightarrow \mathbb{R}$ be a (Lipschitz) continuous function that vanishes if and only if $\rho_{0}$ changes phase. We use for $x \in \mathbb{R}$ the (signed) distance function

$$
\varphi_{0}(x):=\operatorname{sgn}\left(\rho_{0}(x)-\rho_{\min }^{\text {ellipt }}\right) \min \left\{|x-z| \mid \rho_{0} \text { has a phase transition in } z \in \mathbb{R}\right\} .
$$

This and some definitions below require regularity properties for $\mathbf{w}$ which are assumed to hold in this motivation for simplicity. Let $V: \mathbb{R} \times(0, \infty) \rightarrow \mathbb{R}$ be a function that coincides with the velocity of a phase transition at the location of phase transitions. Then the motion of phase boundaries is tracked by the solution $\varphi: \mathbb{R} \times[0, \infty)$ of

$$
\partial_{t} \varphi+V\left|\partial_{x} \varphi\right|=0 \text { in } \mathbb{R} \times(0, \infty), \quad \varphi(\cdot, 0)=\varphi_{0} \text { in } \mathbb{R} .
$$

In our numerical algorithm a discrete version (56) is used at each time step to keep track of the location of phase boundaries. For one space dimension this could also be done "by hand" if the transportation speeds of the phase transitions are given, however we will work with the level-set equation because this concept can be transfered to multiple space dimensions without conceptional problems [27].

Before we present the numerical algorithm (as two sub-algorithms) let us introduce some notations. For $h>0$ we consider an uniform spatial grid with $x_{j}:=j h$ and cells $I_{j}:=\left[x_{j}-h / 2, x_{j}+h / 2\right), j \in \mathbb{Z}$. To start our algorithm we have to assume that the initial data (54) is such that for $j \in \mathbb{Z}$ we have

$$
\boldsymbol{w}_{j}^{0}:=\frac{1}{h} \int_{I_{j}} \boldsymbol{w}_{0}(x) \mathrm{d} x \in \mathcal{A} \times \mathbb{R} .
$$

Algorithm 4.1 (Time evolution). Let (54), (57) be satisfied and define

$$
\varphi_{j+1 / 2}^{0}:=\varphi_{0}\left(x_{j+1 / 2}\right) \quad(j \in \mathbb{Z}) .
$$


The numerical approximation $\boldsymbol{w}_{h}=\left(\rho_{h}, m_{h}\right)^{T}: \mathbb{R} \times\left[0, t^{N}\right) \longrightarrow \mathcal{A} \times \mathbb{R}$ of the weak solution $\mathbf{w}$ is given by

$$
\boldsymbol{w}_{h}(x, t):=\boldsymbol{w}_{j}^{n}, \quad(x, t) \in I_{j} \times\left[t^{n}, t^{n+1}\right) .
$$

The number $N \in \mathbb{N}$, the cell averages $\left\{\boldsymbol{w}_{j}^{n}\right\}_{j \in \mathbb{Z}, n=1, \ldots, N}$, the auxiliary values $\left\{\varphi_{j+1 / 2}^{n}\right\}_{j \in \mathbb{Z}, n=1, \ldots, N}$, and $t^{0}:=$ $0, t^{1}, \ldots, t^{N-1}$ are constructed below in steps (1)-(5) where we use the notation

$$
\mathcal{I}_{\text {liq }}^{n}:=\left\{j \in \mathbb{Z} \mid\left(\varphi_{j-1 / 2}^{n}+\varphi_{j+1 / 2}^{n}\right) \geq 0\right\} \text { and } \mathcal{I}_{\text {vap }}^{n}:=\left\{j \in \mathbb{Z} \mid\left(\varphi_{j-1 / 2}^{n}+\varphi_{j+1 / 2}^{n}\right)<0\right\} \quad(n=0, \ldots, N) .
$$

(1) (Splitting to two single phases, Fig. 11(a).) Suppose at time $t^{n}$ the values $\left\{\boldsymbol{w}_{j}^{n}\right\}_{j \in \mathbb{Z}}$ and $\left\{\varphi_{j+1 / 2}^{n}\right\}_{j \in \mathbb{Z}}$ are given, such that for $j \in \mathbb{Z}$ we have $\rho_{j}^{n} \in \mathcal{A}$. Define

$$
\begin{aligned}
\boldsymbol{w}_{v a p_{j}}^{n}:=\left(\rho_{v a p_{j}}^{n}, m_{v a p_{j}}^{n}\right)^{T}:=\left(\rho_{j}^{n}, m_{j}^{n}\right)^{T} \quad \text { for } j \in \mathcal{I}_{v a p}^{n}, \\
\boldsymbol{w}_{l i q_{j}^{n}}{ }^{n}=\left(\rho_{l i q_{j}^{n}}{ }^{n}, m_{l i q_{j}}^{n}\right)^{T}:=\left(\rho_{j}^{n}, m_{j}^{n}\right)^{T} \quad \text { for } j \in \mathcal{I}_{l i q}^{n} .
\end{aligned}
$$

(2) (Completion of single phase values.) Define ghost values by

$$
\begin{array}{rll}
\boldsymbol{w}_{v a p_{j}}{ }^{n}:=\left(\rho_{v a p_{j}}^{g h}, m_{v a p_{j}}^{g h}{ }^{n}\right. & \text { for } j \in \mathcal{I}_{l i q}^{n}, \\
\boldsymbol{w}_{l i q_{j}}{ }^{n}:=\left(\rho_{l i q_{j}}^{g h^{n}}, m_{l i q_{j}}^{g h^{n}}\right)^{T} & \text { for } j \in \mathcal{I}_{v a p}^{n} .
\end{array}
$$

The ghost values will be given in Algorithm 4.3 below.

(3) (Evolution for single phases and level set, Figs. 11(c) and 11(d).) For $j \in \mathbb{Z}$ set

$$
\begin{aligned}
\boldsymbol{w}_{l i q / v a p_{j}}^{n+1} & :=\boldsymbol{w}_{\text {liq/vap }_{j}}^{n}-\frac{\Delta t^{n}}{h}\left[\boldsymbol{g}_{l i q / v a p}\left(\boldsymbol{w}_{l i q / v a p_{j}}^{n}, \boldsymbol{w}_{l i q / v a p_{j+1}}^{n}\right)-\boldsymbol{g}_{l i q / v a p}\left(\boldsymbol{w}_{l i q / v a p_{j-1}}^{n}, \boldsymbol{w}_{l i q / v a p}{ }_{j}^{n}\right)\right], \\
\varphi_{j+1 / 2}^{n+1} & :=\varphi_{j+1 / 2}^{n}-\Delta t^{n}\left(\frac{V_{j}^{n}+V_{j+1}^{n}}{2} \frac{\varphi_{x}^{-}+\varphi_{x}^{+}}{2}+\alpha_{j+1 / 2} \frac{\varphi_{x}^{-}-\varphi_{x}^{+}}{2}\right) .
\end{aligned}
$$

Here $\boldsymbol{g}_{\text {liq }}:\left(\left(\rho_{\text {liquid }}^{M}, 1 / b\right) \times \mathbb{R}\right)^{2} \rightarrow \mathbb{R}^{2}, \boldsymbol{g}_{\text {vap }}:\left(\left(0, \rho_{\text {vapor }}^{M}\right) \times \mathbb{R}\right)^{2} \rightarrow \mathbb{R}^{2}$ are chosen as consistent numerical flux functions for (1) in the vapor/liquid phase. The local velocities $V_{j}^{n}$ will be given in Algorithm 4.3 below. For the approximation of (56) we use

$$
\varphi_{x}^{+}:=\frac{1}{h}\left(\varphi_{j+3 / 2}^{n}-\varphi_{j+1 / 2}^{n}\right), \quad \varphi_{x}^{-}:=\frac{1}{h}\left(\varphi_{j+1 / 2}^{n}-\varphi_{j-1 / 2}^{n}\right), \quad \alpha_{j+1 / 2}:=\max _{u \in I}\left|\frac{V_{j}^{n}+V_{j+1}^{n}}{2} u\right|
$$

with $I:=\left[\min \left\{\varphi_{x}^{-}, \varphi_{x}^{+}\right\}, \max \left\{\varphi_{x}^{-}, \varphi_{x}^{+}\right\}\right]$.

The time step size is defined as $\Delta t^{n}:=\min \left\{\Delta t_{\text {liq }}^{n}, \Delta t_{\text {vap }}^{n}, \Delta t_{H J}^{n}\right\}$, where $\Delta t_{\text {liq }}^{n}$ and $\Delta t_{\text {vap }}^{n}$ are computed by the CFL condition for the single phase flows and $\Delta t_{H J}^{n}$ by the CFL like restriction for Hamilton-Jacobi equations [15,33].

(4) (Reconstruction of unique two phase values.) Let $t^{n+1}:=t^{n}+\Delta t^{n}$ and

$$
\boldsymbol{w}_{j}^{n+1}:=\left\{\begin{aligned}
\boldsymbol{w}_{l i q j}^{n+1}: & j \in \mathcal{I}_{l i q}^{n+1}, \\
\boldsymbol{w}_{v a p_{j}^{n+1}}: & j \in \mathcal{I}_{\text {vap }}^{n+1} .
\end{aligned}\right.
$$

(5) (Admissibility/nucleation check.) If $\rho_{j}^{n+1} \in \mathcal{A}$ holds for all $j \in \mathbb{Z}$ repeat steps (1) to (4) with $n \mapsto n+1$ otherwise exit the algorithm with $N:=n$. 


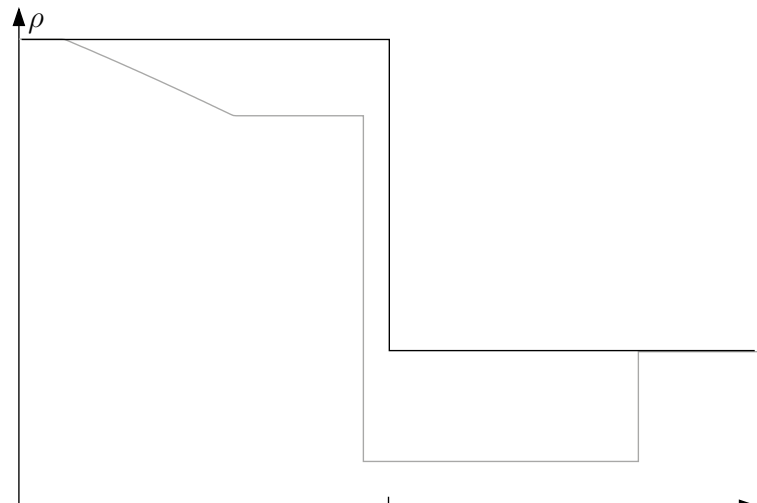

(a) At time $t^{n}$ the density is given. High density values belong to the liquid phase, low ones to the vapor phase. The light grey line denotes the exact solution at later time.

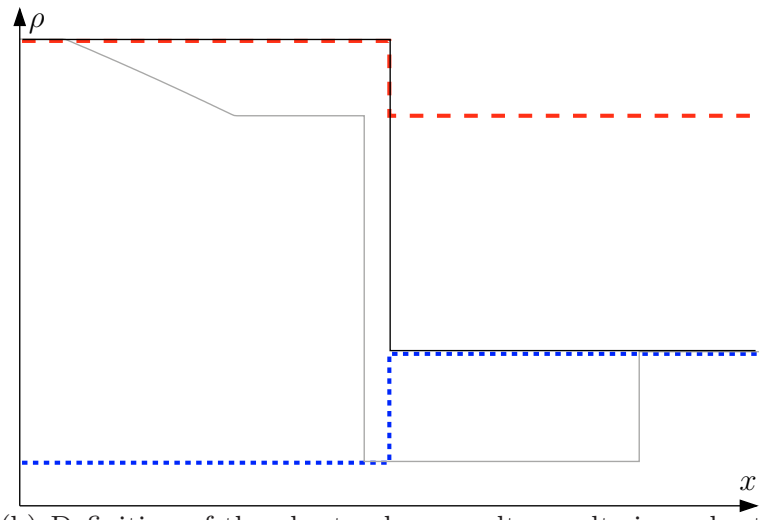

(b) Definition of the ghost values results results in a ghost liquid (red dashed line) and a ghost vapor (blue dotted line) defined in the whole computational domain.

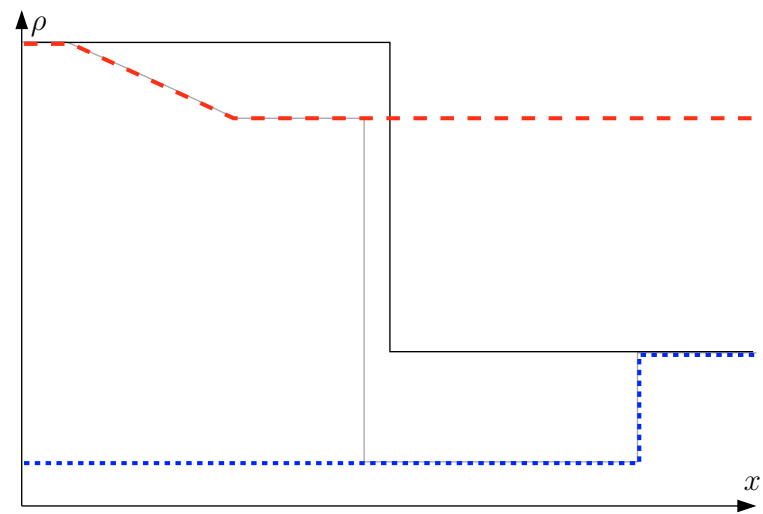

(c) Two-times solution of (1). One for the liquid (red dashed line) and one for the vapor phase (blue dotted line).
$\Lambda \rho$

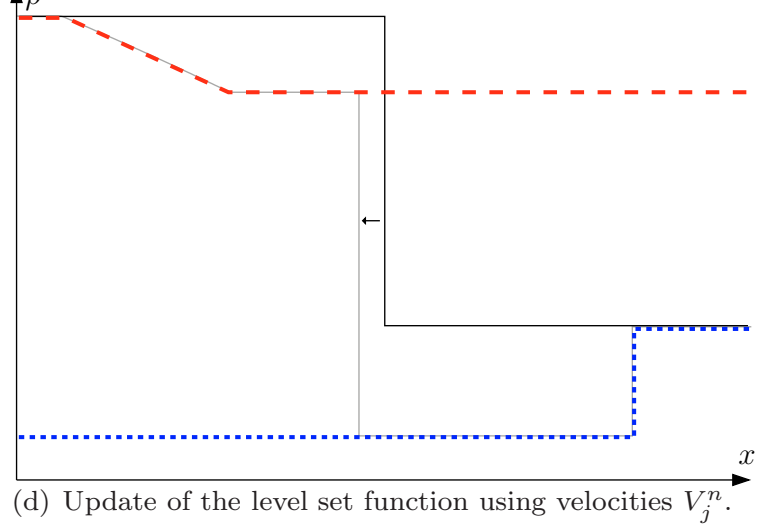

FiguRE 11. Schematic diagrams for the first four steps in Algorithm 4.1.

\section{Remark 4.2.}

- Practically the construction of ghost values in step (2) of Algorithm 4.1 is only necessary in a narrow band about 2-3 elements around points where the discrete level set function changes sign. The implementation can be optimized accordingly.

- In step (3) of Algorithm 4.1 system (1) is solved for two sets of data. However each set belongs to a single phase where (1) is hyperbolic. For the numerical solution one can use the prefered scheme for (isothermal) Euler equations.

If during the calculation there occurs states $\rho_{j}^{n+1}$ within the elliptic region for some $j \in \mathbb{Z}$, we have to exit the algorithm (step (5)). The reason can be a numerical instability. If however schemes are used which preserve the state space (e.g. kinetic schemes) this case indicates that the nucleation of a new phase takes place. Nucleation is not treated in our algorithm but can be easily incorporated by calculating the exact Riemann solutions (according to Thm. 3.6) on the affected elements and construct a new level set function $\varphi$, accordingly. Then, Algorithm 4.1 can be re-entered.

- For the numerical approximation of the solution of the level set equation in step (3) of Algorithm 4.1 we use the scheme as in [33] for Hamilton-Jacobi equations. Of course other choices are possible. 
During the calculation the level set function does not remain a signed distance function as it was initialized. The level set function can steepen or flatten. To avoid numerical errors due to this process we conduct a reinitialization procedure [13,31,35].

Now, we describe the definition of the ghost values in detail as we need them in step (2) of Algorithm 4.1. This is the crucial part of the numerical scheme where we need the solution for the Riemann problem as developed in the first part of the paper.

Algorithm 4.3 (Definition of ghost values). Suppose that for $n \in\{0, \ldots, N-1\}$ the sequences $\left\{\varphi_{j+1 / 2}^{n}\right\}_{j \in \mathbb{Z}}$ and $\left\{\boldsymbol{w}_{l i q / \text { vap }_{j}^{n}}^{n}\right\}_{j \in \mathcal{I}_{l i q / v a p}^{n}}$ are given as defined in step (1) of Algorithm 4.1. The ghost values $\left\{\boldsymbol{w}_{l i q_{j}}^{g h^{n}}\right\}_{j \in \mathcal{I}_{v a p}^{n}}$, $\left\{\boldsymbol{w}_{v a p_{j}}^{g h}\right\}_{j \in \mathcal{I}_{l i q}^{n}}$ for step (2) and the velocities $\left\{V_{j}^{n}\right\}_{j \in \mathbb{Z}}$ for step (3) in Algorithm 4.1 are computed as follows.

(1) (Constant extrapolation, Fig. 12(a).) Define

$$
\boldsymbol{w}_{v a p}^{n}:=\boldsymbol{w}_{v a p}{ }_{\bar{k}}^{n} \quad \text { for } j \in \mathcal{I}_{l i q}^{n},
$$

where $\bar{k}$ is chosen such that $|j-\bar{k}|$ becomes minimal under all values $|j-k|, k \in \mathcal{I}_{\text {vap }}$ and

$$
\boldsymbol{w}_{l i q}^{n} \quad:=\boldsymbol{w}_{l i q}{ }_{\tilde{k}}^{n} \quad \text { for } j \in \mathcal{I}_{v a p}^{n}
$$

with $\tilde{k}$ chosen such that $|j-\tilde{k}|$ becomes minimal under all values $|j-k|, k \in \mathcal{I}_{l i q}$. We obtain two complete sequences $\left\{\boldsymbol{w}_{l i q / v^{\prime} p_{j}}^{n}\right\}_{j \in \mathbb{Z}}$.

(2) (Solving Riemann problems, Fig. 12(b).) For $j \in \mathbb{Z}$ determine (according to Thm. 3.6(ii)) the unique weak solution $\boldsymbol{w}^{(j, n)}: \mathbb{R} \times[0, \infty) \rightarrow \mathcal{A} \times \mathbb{R}$ of the Riemann problems for (1) and initial data

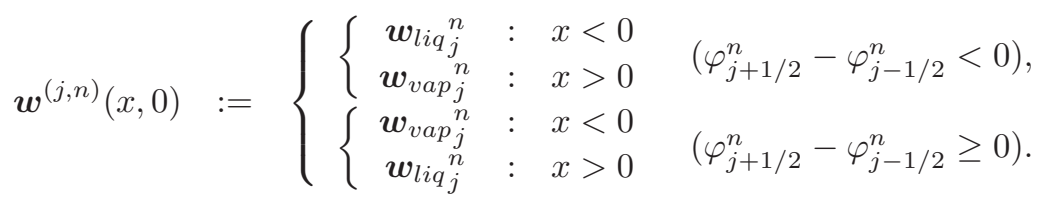

(3) (Definition of ghost values and local phase velocities, Fig. 12(c).) For all $j \in \mathbb{Z}$ the function $\boldsymbol{w}^{(j, n)}$ contains exactly one phase transition which connects two states. For $j \in \mathcal{I}_{\text {vap }}^{n}$ denote the liquid state by $\boldsymbol{w}_{l i q_{j}}^{g h^{n}}$ and, for $j \in \mathcal{I}_{l i q}^{n}$, the vapor state by $\boldsymbol{w}_{v a p_{j}}^{g h}$. For $j \in \mathbb{Z}$ let $V_{j}^{n}$ be given by the phase transition speed.

\section{Remark 4.4.}

(1) To save computing time it is only necessary to solve the Riemann problem in step (2) of Algorithm 4.3 only in a small band around locations of phase transitions. Actually from the Riemann solution we need only the values left and right to a phase transition. One could think of approximations which do not require to resolve all the Riemann pattern in single phases.

(2) The complete algorithm does not preserve conservation of mass and momentum. All numerical experiments we performed show that the loss of exact conservation does not prevent the numerical method to converge for vanishing mesh parameter. We do not present these experiments but they can be found in [27], in particular in Sections 4.4.4 and 4.5.

In view of the fact that up to now there are no convergence proofs even for one-phase flow problems it is not surprising that we are not able to present a convergence result for the general initial data in (54). However for special initial data which lead to a traveling wave solution connecting states in different phases we are able to give the following statement. 


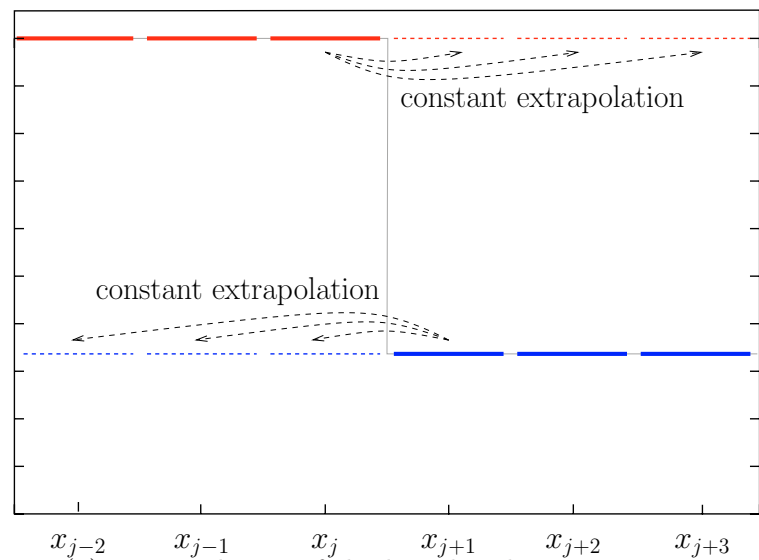

(a) Extrapolation of the liquid and vapor states.

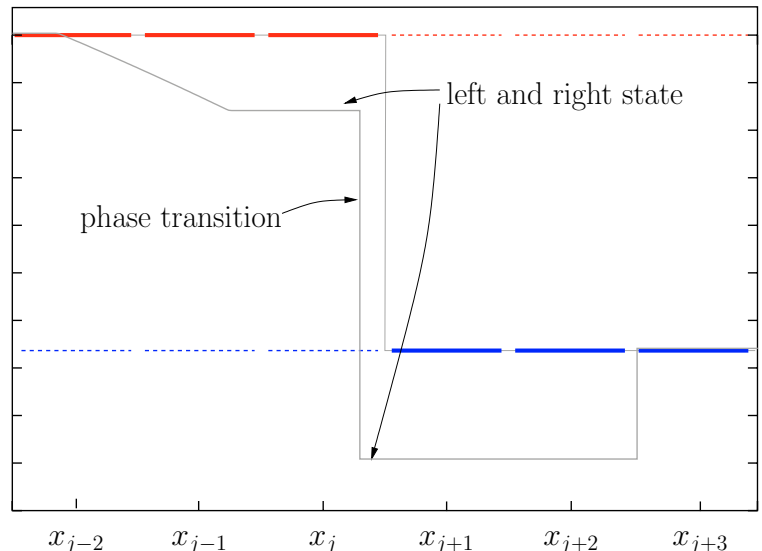

(b) Solution of a Riemann problem for each cell (in this example the same Riemann problem on all cells).

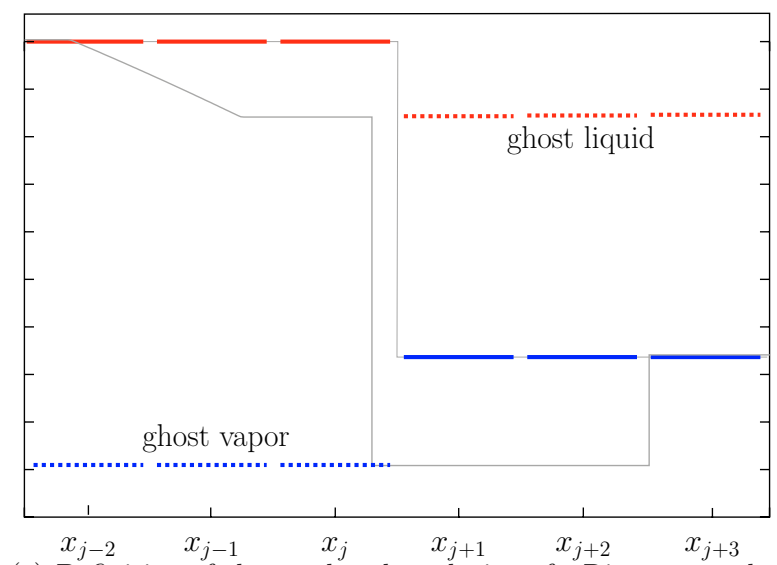

(c) Definition of ghost values by solution of a Riemann problem for each cell.

Figure 12. Schematic diagram of the definition of ghost values by Algorithm 4.3.

Proposition 4.5. Let $h, \Delta t>0$. Assume that $\boldsymbol{w}_{L / R}=\left(\rho_{L / R}, m_{L / R}\right)^{T} \in \mathcal{A} \cup \mathbb{R}$ are given such that $\boldsymbol{w}_{L} \stackrel{p t}{\rightarrow} \boldsymbol{w}_{R}$ is $\Psi$-admissible. Consider the problem (1), (2) for

$$
\boldsymbol{w}_{0}:=\left(\rho_{0}, m_{0}\right)^{T}= \begin{cases}\boldsymbol{w}_{L} & : \quad x-h / 2<0 \\ \boldsymbol{w}_{R} & : \quad x-h / 2>0\end{cases}
$$

According to Theorem 3.6 the unique weak solution is given by

$$
\boldsymbol{w}(x, t)=\left\{\begin{array}{ll}
\boldsymbol{w}_{L}: & : x-V t-h / 2<0, \\
\boldsymbol{w}_{R}: & : x-V t-h / 2>0,
\end{array} \quad V=\frac{m_{R}-m_{L}}{\rho_{R}-\rho_{L}}\right.
$$

Let $\boldsymbol{w}_{h}$ denote the numerical solution obtained by Algorithm 4.1 where we assume that we can solve (56) exactly. Then we have for all $S, t>0$

$$
\left\|\boldsymbol{w}(., t)-\boldsymbol{w}_{h}(., t)\right\|_{L^{1}([-S, S])}=\mathcal{O}(h), \quad \int_{\mathbb{R}} \boldsymbol{w}(x, t)-\boldsymbol{w}_{h}(x, t) \mathrm{d} x=\mathcal{O}(h) .
$$


The function $\boldsymbol{w}_{h}$ takes only $\boldsymbol{w}_{L}$ and $\boldsymbol{w}_{R}$ as values.

The proposition shows in particular that the conservation error scales with the mesh width. Note that the initial phase jump is aligned with the mesh in order to guarantee (57).

Proof. W.l.o.g. we can assume that $\rho_{L}$ is a vapour state and $\rho_{R}$ is a liquid state. From (57), (58), and (60) we conclude by step (1) of Algorithm 4.1

$$
\mathcal{I}_{\text {liq }}^{0}=\{1, \ldots, \infty\}, \quad \mathcal{I}_{\text {vap }}^{0}=\{-\infty, \ldots, 0\}, \quad \boldsymbol{w}_{j}^{0}= \begin{cases}\boldsymbol{w}_{L}: & : j \leq 0 \\ \boldsymbol{w}_{R}: & j>0\end{cases}
$$

Since the states are $\Psi$-admissible we get from step (2) (using Algorithm 4.3) $\boldsymbol{w}_{l i q / v a p}{ }_{j}^{0}=\boldsymbol{w}_{R / L}$ and determine $V_{j}^{0}=V$ for all $j \in \mathbb{Z}$. With the consistency of the numerical flux $g_{l i q / v a p}$ in step (3) and solving the level set equation exactly we obtain $\boldsymbol{w}_{l i q / \text { vap }_{j}}{ }_{1}=\boldsymbol{w}_{R / L}$ and $\varphi_{j+1 / 2}^{1}=\varphi\left(x_{j+1 / 2}\right)=x_{j}-V \Delta t$. Since we have then $\mathcal{I}_{\text {liq }}^{1}=\left\{j \in \mathbb{Z} \mid x_{j-1 / 2}-V \Delta t>0\right\}$ and $\mathcal{I}_{\text {vap }}^{1}=\left\{j \in \mathbb{Z} \mid x_{j-1 / 2}-V \Delta t<0\right\}$ the final update $\boldsymbol{w}_{j}^{1}$ according to step (4) is

$$
\boldsymbol{w}_{j}^{1}= \begin{cases}\boldsymbol{w}_{L} & : \quad x_{j-1 / 2}-V \Delta t \leq 0 \\ \boldsymbol{w}_{R} & : \quad x_{j-1 / 2}-V \Delta t>0\end{cases}
$$

and we can proceed to the next time step. It follows for $n>1$ with induction

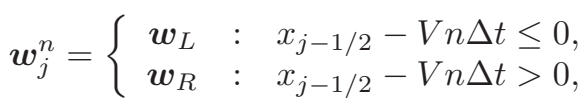

which implies the statement of the proposition taking into account the definition (59) of $\boldsymbol{w}_{h}$ and (61).

\section{NuMERICAL EXPERIMENTS}

In this numerical example we test the new scheme in the presence of a phase transition. Therefore, we solve numerically a Riemann problem with left and right states in different phases. We perform the numerical calculations on the computational space-time domain $[-2,2] \times[0,0.3]$ with different grid widths. To solve the two single phase problems for (1) in Algorithm 4.1 we use as numerical flux the FORCE $E^{4}$ flux [38] with CFL number 0.5. We calculate the $L^{1}$-error and the corresponding experimental convergence rates (EOC).

As initial values according to (3) we choose

$$
\rho_{L}=\rho_{\text {liquid }}^{M}, \quad \rho_{R}=\rho_{\text {vapor }}^{M}, \quad v_{L}=-3.5, \quad v_{R}=0,
$$

with the Maxwell states from (8). The pressure function is as in Remark 1.2. This function satisfies Assumption 1.1 and the exact $\Psi$-admissible solution of the Riemann problem with $\Psi$ from Definition 2.12 is calculated with Theorem 3.6 in the first part of this paper.

\section{Results}

Figure 13 displays the exact solution of the Riemann problem as well as numerical approximations for calculations with different grid sizes at time $T=0.3$. Figures $13(\mathrm{a})$ and 13(c) show the density $\rho$ and velocity $v$ on a fixed mesh with 800 volumes $(h=0.005)$. We first notice, that the complicated structure of the solution, consisting of a small shock wave followed by a (discontinuous) phase transition with attached rarefaction wave and finally an attached wave, is reproduced very well. Also the constant states of the density $\rho$ and velocity $v$ are met quite good. The shock wave on the very left is better resolved than the attached wave on the right, whereas the phase transition is hit perfectly. This demonstrates that the left and right hand states of the phase transition, which define the velocity $V$ in the level set equation (56), are reproduced very well. 


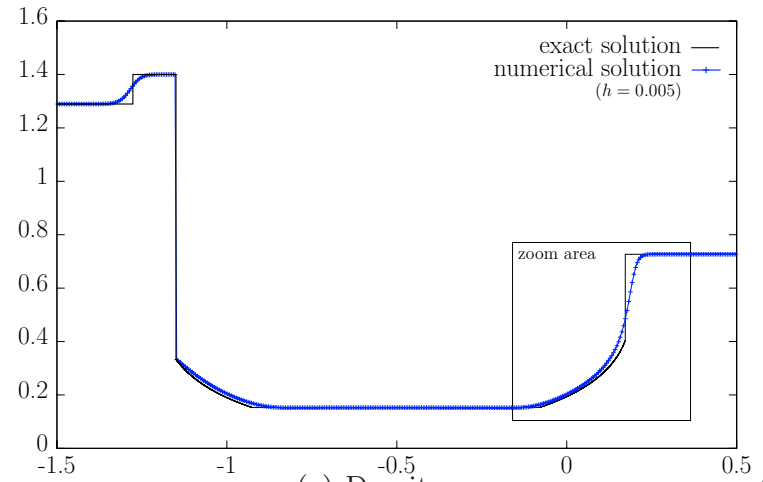

(a) Density $\rho$

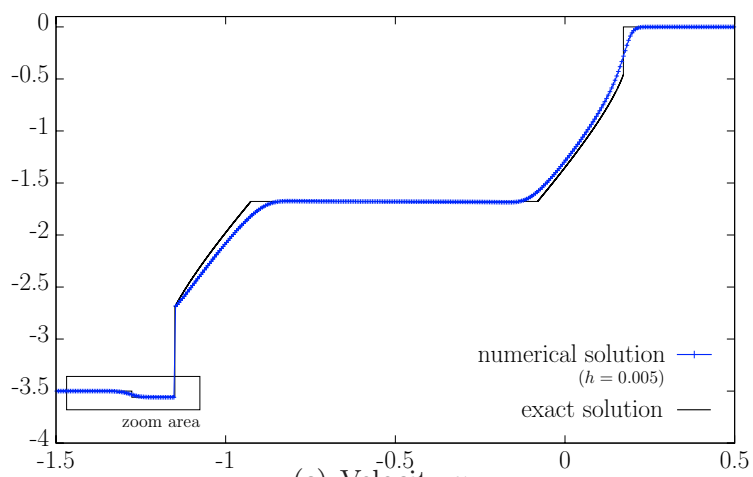

(c) Velocity $v$
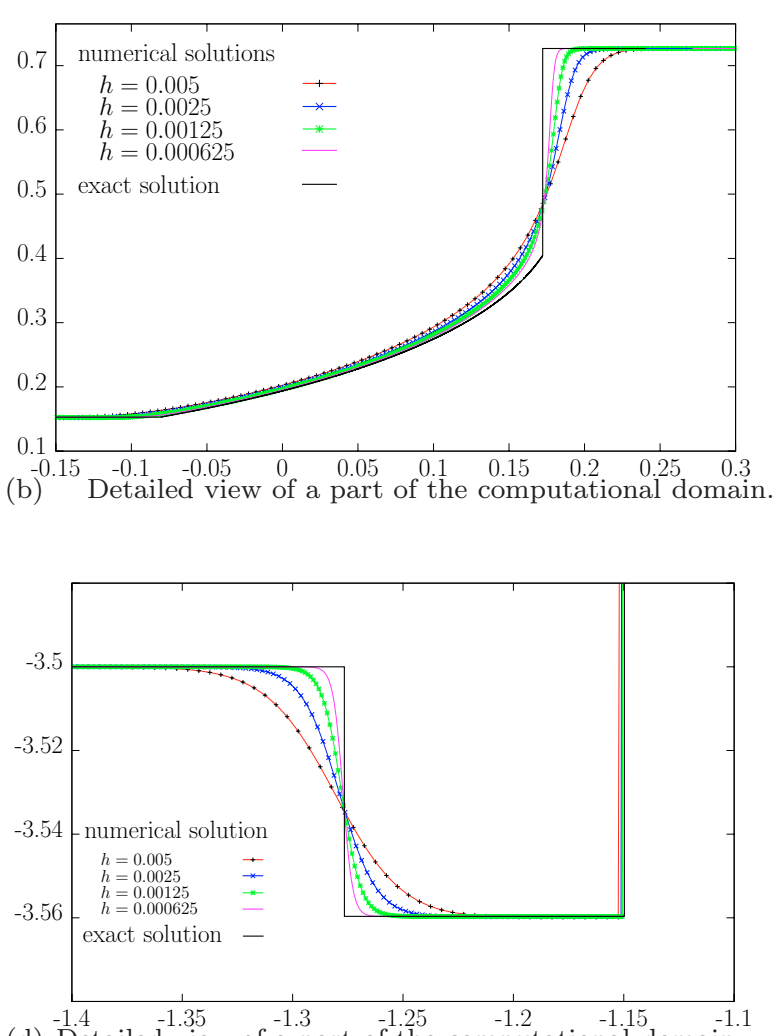

(d) ${ }^{-1.4}$ Detailed view of a part of the computational domain.

FIGURE 13. Graph of exact and approximate solutions for Riemann problem with shock wave, phase transition with attached rarefaction wave and an attached wave in the vapor phase.

TABLE 1. $L^{1}$-error and EOC rate for subsequent refinement levels of the grid.

\begin{tabular}{|l|l|l|l|l|}
\hline Grid size & $L^{1}$-error of $\rho$ & EOC & $L^{1}$-error of $v$ & EOC \\
\hline 0.04 & 0.08953370 & 0.85 & 0.22612267 & 1.03 \\
0.02 & 0.04975303 & 0.48 & 0.11056125 & 0.39 \\
0.01 & 0.03562381 & 0.75 & 0.08429837 & 0.84 \\
0.005 & 0.02126755 & 0.77 & 0.04722471 & 0.88 \\
0.0025 & 0.01247926 & 0.79 & 0.02572075 & 0.90 \\
0.00125 & 0.00721779 & 0.83 & 0.01378924 & 0.97 \\
0.000625 & 0.00406572 & 0.87 & 0.00702086 & 1.08 \\
0.0003125 & 0.00222163 & 0.77 & 0.00332194 & 0.77 \\
0.00015625 & 0.00130720 & 0.77 & 0.00195002 & 0.75 \\
0.000078125 & 0.00076604 & 0.76 & 0.00115614 & 0.70 \\
0.0000390625 & 0.00045315 & 0.85 & 0.00071321 & 0.89 \\
0.00001953125 & 0.00025183 & & 0.00038543 & \\
\hline
\end{tabular}

Figures 13(b) and 13(d) show a detailed view of part of the exact and approximated solutions on different grids with subsequently finer grid size. We notice that the numerical approximations improve as the grid is refined. This indicates convergence of the scheme. 
The convergence of the scheme can be seen in Table 1 where we list the $L^{1}$-error of the numerical approximations on different meshes at time $T=0.3$ for the density $\rho$ as well as for the velocity $v$. We also show the resulting experimental order of convergence. The values of the EOC are around 0.7-0.8. This result is in accordance to the classical Finite Volume scheme for hyperbolic systems.

Summarized we can say that the new scheme for hyperbolic elliptic systems (that can describe phase transitions) converges with about the same rate as a classical Finite Volume scheme.

\section{REFERENCES}

[1] R. Abeyaratne and J. Knowles, Kinetic relations and the propagation of phase boundaries in solids. Arch. Ration. Mech. Anal. 114 (1991) 119-154.

[2] R. Abgrall and S. Karni, Compressible multifluid flows. J. Comput. Phys. 169 (2001) 594-623.

[3] R. Abgrall and R. Saurel, Discrete equations for physical and numerical compressible multiphase mixtures. J. Comput. Phys. 186 (2003) 361-396.

[4] T.D. Aslam, A level set algorithm for tracking discontinuities in hyperbolic conservation laws II: Systems of equations. J. Sci. Comput. 19 (2003) 37-62.

[5] N. Bedjaoui and P.G. LeFloch, Diffusive-dispersive travelling waves and kinetic relations. II. A hyperbolic-elliptic model of phase-transition dynamics. Proc. Roy. Soc. Edinburgh Sect. A 132A (2002) 1-21.

[6] S. Benzoni-Gavage, Stability of multi-dimensional phase transitions in a van der Waals fluid. Nonlinear Anal., Theory Methods Appl. 31 (1998) 243-263.

[7] C. Chalons, Transport-Equilibrium Schemes for Computing Nonclassical Shocks. I. Scalar Conservation Laws. Preprint, Laboratoire Jacques-Louis Lions (2005).

[8] C. Chalons and P.G. LeFloch, Computing undercompressive waves with the random choice scheme. Interfaces Free Bound. 5 (2003) 129-158.

[9] R.M. Colombo and A. Corli, Continuous dependence in conservation laws with phase transitions. SIAM J. Math. Anal. 31 (1999) 34-62.

[10] R.M. Colombo and A. Corli, Stability of the Riemann semigroup with respect to the kinetic condition. Quart. Appl. Math. 62 (2004) 541-551.

[11] C.M. Dafermos, Hyperbolic Conservation Laws in Continuum Physics. Grundlehren der mathematischen Wisenschaften 325. Springer (2000).

[12] H. Fan and M. Slemrod, Dynamic flows with liquid/vapor phase transitions, in Handbook of mathematical fluid dynamics, Vol. I, North-Holland, Amsterdam (2002) 373-420.

[13] R.P. Fedkiw, T. Aslam, B. Merriman and S. Osher, A non-oscillatory Eulerian approach to interfaces in multimaterial flows (the ghost fluid method). J. Comput. Phys. 152 (1999) 457-492.

[14] R.P. Fedkiw, T. Aslam and S. Xu, The ghost fluid method for deflagration and detonation discontinuities. J. Comput. Phys. 154 (1999) 393-427.

[15] E. Godlewski and P.-A. Raviart, Numerical approximation of hyperbolic systems of conservation laws. Appl. Math. Sci. 118 Springer (1996).

[16] E. Godlewski and N. Seguin, The Riemann problem for a simple model of phase transition. Commun. Math. Sci. 4 (2006) $227-247$.

[17] B. Hayes and P.G. LeFloch, Nonclassical shocks and kinetic relations: strictly hyperbolic systems. SIAM J. Math. Anal. 31 (2000) 941-991.

[18] T.Y. Hou, P. Rosakis and P.G. LeFloch, A level-set approach to the computation of twinning and phase-transition dynamics. J. Comput. Phys. 150 (1999) 302-331.

[19] M. Kac, G.E. Uhlenbeck and P.C. Hemmer, On the van der Waals theory of the vapor-liquid equilibrium. I. Discussion of a one-dimensional model. J. Math. Phys. 4 (1963) 216-228.

[20] S. Karni and R. Abgrall, Ghost-Fluids for the Poor: A Single Fluid Algorithm for Multifluids, in Lecture Notes in Mathematics, Proceedings of the 10th International Conference on Hyperbolic problems, theory and numerics, Springer (2001) 293-302.

[21] O. Le Métayer, J. Massoni and R. Saurel, Modelling evaporation fronts with reactive Riemann solvers. J. Comput. Phys. 205 (2005) 567-610.

[22] P.G. LeFloch, Propagating phase boundaries: Formulation of the problem and existence via the Glimm method. Arch. Ration. Mech. Anal. 123 (1993) 153-197.

[23] P.G. LeFloch, Hyperbolic Systems of Conservation Laws: The Theory of Classical and Nonclassical Shock Waves. Lectures in Mathematics. ETH Zürich, Birkhäuser (2002).

[24] P.G. LeFloch and M.D. Thanh, Nonclassical Riemann solvers and kinetic relations. II. An hyperbolic-elliptic model of phase transitions. Proc. Royal Soc. Edinburgh A 131A (2001) 1-39.

[25] P.G. LeFloch, J.M. Mercier and C. Rohde, Fully discrete, entropy conservative schemes of arbitrary order. SIAM J. Numer. Anal. 40 (2002) 1968-1992. 
[26] T.G. Liu, B.C. Khoo and K.S. Yeo, Ghost fluid method for strong impacting on material interfaces. J. Comput. Phys. 190 (2003) 651-681.

[27] C. Merkle, Dynamical Phase Transitions in Compressible Media. Doctoral dissertation, Albert-Ludwigs-Universität Freiburg (2006) http://www.freidok.uni-freiburg.de/volltexte/2674/.

[28] C. Merkle and C. Rohde, Computation of dynamical phase transitions in solids. Appl. Numer. Math. 56 (2006) $1450-1463$.

[29] W. Mulder, S. Osher and J. Sethian, Computing interface motion in compressible gas dynamics. J. Comput. Phys. 100 (1992) 209-228.

[30] S. Müller and A. Voß, The Riemann problem for the Euler equations with nonconvex and nonsmooth equation of state: construction of wave curves. SIAM J. Sci. Comput. 28 (1992) 651-681.

[31] S. Osher and R. Fedkiw, Level Set Methods and Dynamic Implicit Surfaces. Appl. Math. Sci. 153. Springer (2003).

[32] S. Osher and J. Sethian, Fronts propagating with curvature dependent speed: Algorithms based on Hamilton-Jacobi formulations. J. Comput. Phys. 79 (1988) 12-49.

[33] S. Osher and C.-W. Shu, High-order essentially nonoscillatory schemes for Hamilton-Jacobi equations. SIAM J. Numer. Anal. 28 (1991) 907-922.

[34] D. Peng, B. Merriman, S. Osher, H.K. Zhao and M. Kang, A PDE-based fast local level set method. J. Comput. Phys. 155 (1999) 410-438.

[35] G. Russo and P. Smereka, A remark on computing distance functions. J. Comput. Phys. 163 (2000) 51-67.

[36] D. Serre, Systems of Conservation Laws 1. Cambridge University Press (1999).

[37] M. Sussman, P. Smereka and S. Osher, A level set approach for computing solutions to incompressible two-phase flow. J. Comput. Phys. 114 (1994) 146-154.

[38] E.F. Toro, Multi-Stage Predictor-Corrector Fluxes for Hyperbolic Equations. Technical Report NI03037-NPA Isaac Newton Institute for Mathematical Sciences (2003).

[39] L. Truskinovsky, Kinks versus Shocks, in Shock induced transitions and phase structures in general media, Springer, New York (1993) 185-229.

[40] L. Truskinovsky and A. Vainchtein, Explicit kinetic relation from "first principles", in Mechanics of material forces 11, Advances in Mechanics and Mathematics, P. Steinmann and G.A. Maugin (Eds.), Springer (2005) 43-50.

[41] X. Zhong, T.Y. Hou and P.G. LeFloch, Computational method for propagating phase boundaries. J. Comput. Phys. 124 (1996) 192-216. 